\title{
Spatial Modulation Aided Sparse Code-Division Multiple Access
}

\author{
Yusha Liu, Student Member, IEEE, Lie-Liang Yang, Fellow, IEEE, and Lajos Hanzo, Fellow, IEEE
}

\begin{abstract}
In order to support high-user-load multiple-access (MA), we propose a non-orthogonal MA scheme based on a beneficial amalgam of spatial modulation (SM) and sparse codedivision multiple-access (SCDMA), which is termed as the SMSCDMA. Hence, SM-SCDMA inherits both the merits of SM with single radio-frequency MIMO transceiver implementation and the advantages of SCDMA relying on low-complexity signal detection. In this paper, we evaluate the potential of SM-SCDMA as well as its low-complexity near-optimum signal detection. Given these objectives, we consider both the maximum likelihood detection (MLD) and the message passing algorithm aided detection (MPAD) that is derived based on the maximum $a$ posteriori principles. In order to evaluate the performance of large SM-SCDMA without relying on time-consuming simulations, we propose new approaches for analyzing the performance of SM-SCDMA systems. A range of formulas that are valid in the signal-to-noise ratio (SNR) region of practical interest are derived. Finally, the performance of SM-SCDMA systems is investigated by addressing diverse design concerns. Our studies and performance results show that SM-SCDMA constitutes a promising MA scheme for the future ultra dense systems. Assisted by the MPAD, it is capable of supporting high-user-load MA transmission associated with a normalized user-load factor of two.
\end{abstract}

Index Terms-CDMA, NOMA, spatial modulation, low density signature, maximum a posteriori detection, maximum-likelihood detection, message passing algorithm, performance analysis.

\section{INTRODUCTION}

During the 2010s, research interests in multiuser communications have been focused on non-orthogonal multiple-access (NOMA) [1-15], with the motivation of supporting ultra-dense deployment, which faces the problem of supporting more users (or devices) than the number of resource units available. For uplink communications, sparse code spread CDMA [16, 17], or simply termed as sparse CDMA (SCDMA), has been proposed for meeting these objectives. In SCDMA, each spreading sequence contains only a small number of non-zero entries. Hence, each user's transmitted symbol is represented by a small number of non-zero chips in the spreading codes. Within this family, the proposed schemes include both low density signature (LDS) assisted CDMA [2] and sparse code multiple access (SCMA) [18-21]. The studies in [2,18] show that by exploiting the sparsity of the signatures, SCDMA becomes capable of supporting more users than conventional CDMA [22], while relying on relatively low-complexity detectors. The reason behind this is that the employment of low density signature codes allows us to invoke a message passing algorithm

Y. Liu, L.-L. Yang and L. Hanzo are with School of Electronics and Computer Science, University of Southampton, SO17 1BJ, UK. (E-mail: yl6g15, lly, lh@ecs.soton.ac.uk). The author would like to acknowledge with thanks the financial assistance from EPSRC of UK and ERC of EU.
(MPA) [23-26] assisted detector for achieving near-optimum performance. By contrast, in the conventional CDMA systems, each user's transmitted symbol is spread by fully populated spreading codes, hence it is difficult to design practical lowcomplexity near-optimum detection schemes, especially, when supporting a high normalized user-load.

As a parallel development in multiple-input multiple-output (MIMO) systems, spatial modulation (SM) has drawn a lot of research attention in recent years, as evidenced by [2729] and the references therein. These schemes include spaceshift keying (SSK) [30,31] conveying information solely by the indices of the transmit antennas (TAs). The SM of [27, 30, 32] activates one out of several TAs to convey a classic amplitudephase modulation (APM) symbol, such as, quadrature amplitude modulated (QAM) symbol. By contrast, generalized $S M$ (GSM) [33] simultaneously activates multiple TAs to convey multiple APM symbols, while space-time shift-keying (STSK) [34] exploits all degrees of freedom in the joint spacetime domain for information delivery. Additionally, owing to the recent research interest in NOMA, the authors in [15] have proposed and investigated a NOMA-based SM scheme. Similarly, by activating one or a subset of receive antennas with the aid of appropriate transmitter preprocessing, a range of preprocessing assisted SM (PSM) schemes have been proposed. These include the preprocessing-aided SSK [35] solely using receive antenna indices for implicit information delivery, and the PSM of [35] that conveys information by activating a single receive antenna out of several for conveying an APM symbol. By contrast, the generalized PSM (GPSM) of [36] simultaneously activates multiple receive antennas and conveys multiple APM symbols, etc. Various extensions of the above-mentioned SM or PSM schemes have also been surveyed in [28, 29].

Multiple-access (MA) communications has also been studied in association with SM [37-43]. However, in these SMassisted MA schemes, users are supported solely by the spatial-domain resources, which may require an excessive number of receive antennas at a base-station (BS) [38, 4043]. This is because the number of BS antennas should linearly increase with the number of users, in order to guarantee the required quality-of-services (QoS) by employing practically implementable detectors at the BS. Explictly, the implementation of these schemes faces challenges in terms of antenna deployment, channel estimation, pilot-overhead, etc. This situation becomes even severer, when a future MA system has to support a huge number of communication devices. In this case, SM amalgamated with other MA techniques [44$46]$ is more desirable for practical implementations. Therefore, 
we intrinsically amalgamate SM and NOMA, resulting in our Spatial Modulated Sparse CDMA (SM-SCDMA) scheme conceived for supporting MA communications in heavily loaded communications environments. In summary, the contributions of the paper are as follows:

- A SM-SCDMA scheme is proposed for supporting a high normalized user-load in uplink communications. We assume that each user employs a few TAs for supporting the SM, while the BS employs several receive antennas for enhancing the detection reliability, where MA is achieved with the aid of SCDMA. Since our SM-SCDMA relies on the principle of NOMA, it has the potential of supporting the MA transmission, where the number of (active) users is higher than the total number of chips in the spreading codes.

- It has been recognized that employing SM is beneficial for the implementation of MIMO using a low number of radio-frequency transceivers, and provides lower complexity receiver implementation options [27, 28]. However, when there is a high number of users sharing the resources based on CDMA, the design of relatively lowcomplexity detectors that are capable of achieving nearoptimum performance is challenging. In this paper, we first consider the classic maximum-likelihood detection (MLD) in order to study the best possible potential of the SM-SCDMA scheme. Then, we derive a maximum $a$ posteriori detector (MAPD), based on which we develop a reduced complexity MPA-aided detector (MPAD) that is suitable for SM-SCDMA systems, which employs SM and exploits receiver diversity.

- In most existing references on LDS-CDMA or SCMA, the performance study depends mainly on simulations, which can typically deal with tens of users. However, it is hard to simulate the performance of a MA system supporting hundreds or even thousands of users. However, future NOMA systems are expected to support a huge number of users, hence it is interesting to predict the achievable performance of such large-scale MA systems. With this motivation, in this paper, we mathematically analyze the error performance of SM-SCDMA systems by first deriving the single-user bit error rate (BER) bound and then analyzing the approximate BER of multi-user SM-SCDMA systems. By exploiting the specific structure of SM-SCDMA, a range of formulas are derived, which allow us to estimate the BER performance of the SMSCDMA systems of arbitrary size. Note that in [47], the authors have analyzed the pairwise error probability (PEP) and upper-bound average symbol error rate (ASER) of the general multidimensional constellations with MLD, and considered the LDS-CDMA and SCMA as special cases. By contrast, the authors of [48] have derived a lower bound for the ASER of the SCMA.

- The performance of SM-SCDMA systems is investigated both by Monte-Carlo simulations and by the evaluation of the formulas derived. Based on the SM-SCDMA systems of relatively small size, we verify the formulas derived by simulation and find their valid ranges. Then, the performance of relatively large-scale SM-SCDMA systems is investigated based on the numerical evaluation of our formulas derived. Furthermore, we address the impact of the related parameters on the BER performance attained, and demonstrate the efficiency of the MPAD.

- Finally, we propose a novel 8QAM scheme. When communicating over Gaussian channels, it outperforms all the existing 8QAM schemes in the relatively low signalto-noise $(\mathrm{SNR})(\leq 10 \mathrm{~dB})$ region, while achieving a similar BER performance to the best existing 8QAM schemes in the relatively high-SNR region. By contrast, when communicating over Rayleigh fading channels, it outperforms all the existing 8QAM schemes within the SNR region considered.

The rest of this paper is structured as follows. Section II describes the proposed SM-SCDMA system model. Then, the detection algorithms are addressed in Section III. Section IV analyzes the BER of the SM-SCDMA system, while the BER performance of SM-SCDMA systems is investigated in Section V. Finally, Section VI summarizes the main conclusions of our research.

\section{SM-SCDMA SYSTEM MODEL}

In this section, we describe the transmitter and receiver models in Sections II-A and II-B, respectively. Furthermore, our main assumptions and notations are detailed.

\section{A. Transmitter Model}

Let us consider a single-cell wireless uplink, where $K$ users simultaneously transmit their information to a single BS. In order to avoid dealing with the trivial cases, but to focus our attention on the important principles, we assume that all users employ the same $M_{1}$ number of TAs satisfying $M_{1}=2^{b_{1}}$, where $b_{1}$ is an integer. We assume that the BS employs $U$ receive antennas for attaining receiver diversity. Based on the principles of SM [27], we assume that within a symbol duration of $T_{s}$ seconds, one of the $M_{1}$ TAs of a user is activated for transmitting $b_{1}$ bits, forming an $M_{1}$ SSK scheme [31]. Hence, the SSK symbol set can be expressed as $\mathcal{S}_{1}=\left\{0,1, \ldots, M_{1}-1\right\}$ and the corresponding $b_{1}$-bit symbols can be their natural binary representations. In addition to the $M_{1} \mathrm{SSK}$, the SM also uses the activated TA to transmit $b_{2}=\log _{2} M_{2}$ bits, relying on a $M_{2}$-ary APM ( $\left.M_{2} \mathrm{APM}\right)$, such as $M_{2}$-ary QAM $\left(M_{2} \mathrm{QAM}\right)$. For convenience, the APM symbol set is expressed as $\mathcal{S}_{2}=\left\{s_{2,0}, s_{2,1}, \ldots, s_{2, M_{2}-1}\right\}$, whose elements satisfy $\sum_{i=0}^{M_{2}-1}\left|s_{2, i}\right|^{2} / M_{2}=1$. Therefore, when considering both the $M_{1}$ SSK and $M_{2}$ APM, $b=\left(b_{1}+b_{2}\right)$ bits per symbol can be transmitted by a user to the BS. Note that the SM model considered can be readily extended to the generalized SM model of [28], where multiple TAs are activated for simultaneously transmitting multiple APM symbols.

Fig. 1 depicts the transmitter schematic of the $k$ th user of the SM-SCDMA system. During a symbol period, the $b$-bit symbol $\boldsymbol{b}_{k}$ of user $k$ is first partitioned into two sub-symbols, the SSK symbol $\boldsymbol{b}_{k 1}$ consisting of the first $b_{1}$ bits and the APM symbol $\boldsymbol{b}_{k 2}$ consisting of the remaining $b_{2}$ bits. Let $s_{k 1} \in \mathcal{S}_{1}$ 


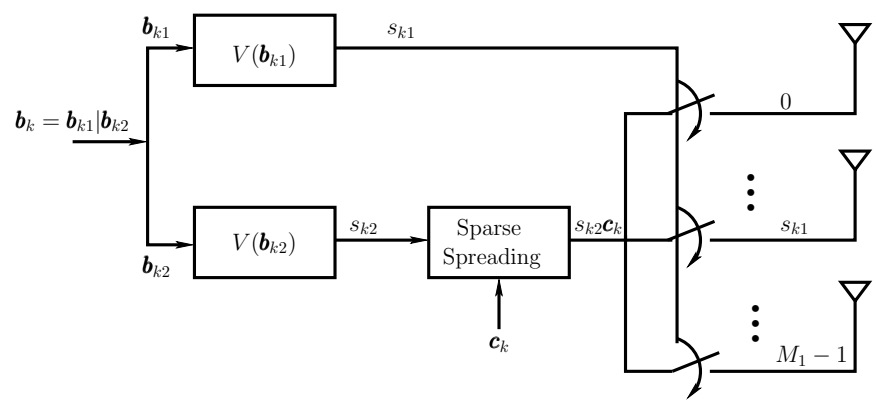

Fig. 1. Transmitter schematic diagram of the $k$ th user in the SM-SCDMA system.

be an integer value obtained from the mapping $V_{1}\left(\boldsymbol{b}_{k 1}\right)$, which determines the TA activated by user $k$. Furthermore, let $s_{k 2} \in \mathcal{S}_{2}$ be obtained from the mapping $V_{2}\left(\boldsymbol{b}_{k 2}\right)$, which is an APM symbol. Following the principles of SCDMA [2], $s_{k 2}$ is spread to a maximum of $d_{x}$ chips $\left(d_{x}<<N\right)$ using the spreading sequence $\boldsymbol{c}_{k}=\left[c_{k 0}, c_{k 1}, \ldots, c_{k(N-1)}\right]^{T}$ assigned to user $k$, where $\boldsymbol{c}_{k}$ is of $N$-length and has $d_{x}$ non-zero chips, satisfying $\left\|\boldsymbol{c}_{k}\right\|^{2}=1$. Furthermore, we assume that the maximum number of users sharing any of the $N$ chips is $d_{f}$. In the following analysis, for simplicity, we assume that $d_{x}$ and $d_{f}$ are constants. The set of sparse codes achieving these are referred to as the regular sparse codes. Finally, as shown in Fig. 1, the $k$ th user's SM-SCDMA signal is transmitted from the $s_{k 1}$ th TA, following some further transmitter processing that is not shown in the figure.

Therefore, given the SM symbol $s_{k 1} \mid s_{k 2}$ of user $k$, the unityenergy baseband equivalent signal transmitted by user $k$ can be expressed as

$$
\boldsymbol{t}_{k}\left(s_{k 1}\right)=s_{k 2} \boldsymbol{c}_{k}, \quad k=1,2, \cdots, K
$$

where $\boldsymbol{t}_{k}\left(s_{k 1}\right)$ indicates that the signal is transmitted by the $s_{k 1}$ th antenna. For convenience of description, below we define $\mathcal{X}=\mathcal{S}_{1} \otimes \mathcal{S}_{2}$, which is a set containing the $M\left(=M_{1} M_{2}\right)$ combinations of the elements in $\mathcal{S}_{1}$ with those in $\mathcal{S}_{2}$.

\section{B. Receiver Model}

Assume that the channel between any user and the BS experiences flat Rayleigh fading ${ }^{1}$. Let the channel impulse response (CIR) between the $s_{k 1}$ th TA of user $k$ and the $U$ receive antennas at the $\mathrm{BS}$ be expressed as

$$
\boldsymbol{h}_{s_{k 1}}=\left[h_{s_{k 1}}^{(0)}, h_{s_{k 1}}^{(1)}, \cdots, h_{s_{k 1}}^{(U-1)}\right]^{T}, s_{k 1}=0, \ldots, M_{1}-1
$$

where $h_{s_{k 1}}^{(u)}$ are independent identically distributed (iid) complex Gaussian random variables with zero mean and a variance of 0.5 per dimension. Therefore, when there are $K$ uplink users, the $N$-length observation vector received by the $u$ th receive antenna is given by

$$
\begin{gathered}
\boldsymbol{y}_{u}=\sum_{k=1}^{K} h_{s_{k 1}}^{(u)} \boldsymbol{t}_{k}\left(s_{k 1}\right)+\boldsymbol{n}_{u}=\sum_{k=1}^{K} h_{s_{k 1}}^{(u)} s_{k 2} \boldsymbol{c}_{k}+\boldsymbol{n}_{u}, \\
\quad u=0,1, \cdots, U-1
\end{gathered}
$$

${ }^{1}$ Frequency-selective fading may be eliminated by multicarrier SMSCDMA systems. where $\boldsymbol{n}_{u}$ is the Gaussian noise vector of zero mean and having a covariance matrix of $2 \sigma^{2} \boldsymbol{I}_{N}$, expressed as $\mathcal{C N}\left(0,2 \sigma^{2} \boldsymbol{I}_{N}\right)$, with $\sigma^{2}=1 /\left(2 \gamma_{0}\right)$, and $\gamma_{0}=b \gamma_{b}$ being the SNR per symbol, while $\gamma_{b}$ the SNR per bit.

Let $\boldsymbol{y}=\left[\boldsymbol{y}_{0}^{T}, \boldsymbol{y}_{1}^{T}, \cdots, \boldsymbol{y}_{U-1}^{T}\right]^{T}$ and $\boldsymbol{n}=$ $\left[\boldsymbol{n}_{0}^{T}, \boldsymbol{n}_{1}^{T}, \cdots, \boldsymbol{n}_{U-1}^{T}\right]^{T}$. Then, we can express $\boldsymbol{y}$ as

$$
\boldsymbol{y}=\sum_{k=1}^{K}\left(\boldsymbol{I}_{U} \otimes \boldsymbol{c}_{k}\right) \boldsymbol{h}_{s_{k 1}} s_{k 2}+\boldsymbol{n},
$$

where $\otimes$ denotes the Kronecker product [49]. Furthermore, we can rewrite (4) in a compact form as

$$
\boldsymbol{y}=\boldsymbol{H}\left(\boldsymbol{s}_{1}\right) \boldsymbol{s}_{2}+\boldsymbol{n}
$$

where $\boldsymbol{H}\left(\boldsymbol{s}_{1}\right)=\left[\left(\boldsymbol{I}_{U} \otimes \boldsymbol{c}_{1}\right) \boldsymbol{h}_{s_{11}}, \cdots,\left(\boldsymbol{I}_{U} \otimes \boldsymbol{c}_{K}\right) \boldsymbol{h}_{s_{K 1}}\right], \boldsymbol{s}_{1}=$ $\left[s_{11}, s_{21}, \ldots, s_{K 1}\right]^{T}$ collects the SSK symbols and $\boldsymbol{s}_{2}=$ $\left[s_{12}, s_{22}, \ldots, s_{K 2}\right]^{T}$ collects the APM symbols transmitted by the $K$ users.

\section{Signal Detection}

In this section, we address the detection of SM-SCDMA signals. We first consider the optimal MLD in Section III-A, whereas Section III-B derives the MAPD. Then, based on this the MPAD is developed in Section III-C.

\section{A. Maximum-Likelihood Detection (MLD)}

Let us express the symbols transmitted by the $K$ users as a vector $\boldsymbol{x}=\left[x_{1}, x_{2}, \cdots, x_{K}\right]^{T}$, where we express $x_{k}=s_{k 1} \mid s_{k 2}, x_{k} \in \mathcal{X}$. We assume that the BS employs the knowledge of both the channels and of the spreading sequences. Then, the MLD finds the estimate of $x$ by solving the optimization problem of ${ }^{2}$

$$
\begin{aligned}
\hat{\boldsymbol{x}}= & \arg \min _{\tilde{\boldsymbol{x}} \in \mathcal{X}^{K}}\left\{\left\|\boldsymbol{y}-\sum_{k=1}^{K}\left(\boldsymbol{I}_{U} \otimes \boldsymbol{c}_{k}\right) \boldsymbol{h}_{\tilde{s}_{k 1}} \tilde{s}_{k 2}\right\|^{2}\right\} \\
= & \arg \max _{\tilde{\boldsymbol{x}} \in \mathcal{X}^{K}}\left\{\sum_{k=1}^{K} \Re\left\{\tilde{s}_{k 2}^{*} \boldsymbol{h}_{\tilde{s}_{k 1}}^{H}\left(\boldsymbol{I}_{U} \otimes \boldsymbol{c}_{k}\right)^{H} \boldsymbol{y}\right\}\right. \\
& \left.-\frac{1}{2} \sum_{k=1}^{K} \sum_{i=1}^{K} \tilde{s}_{k 2}^{*} \boldsymbol{h}_{\tilde{s}_{k 1}}^{H}\left(\boldsymbol{I}_{U} \otimes \boldsymbol{c}_{k}\right)^{H}\left(\boldsymbol{I}_{U} \otimes \boldsymbol{c}_{i}\right) \boldsymbol{h}_{\tilde{s}_{i 1}} \tilde{s}_{i 2}\right\}
\end{aligned}
$$

where $\tilde{x}_{k}=\tilde{s}_{k 1} \mid \tilde{s}_{k 2}$ determines the term $\boldsymbol{h}_{\tilde{s}_{k 1}} \tilde{s}_{k 2}$, and $\Re\{x\}$ returns the real part of $x$. In the above equation, we can show that

$$
\boldsymbol{h}_{\tilde{s}_{k 1}}^{H}\left(\boldsymbol{I}_{U} \otimes \boldsymbol{c}_{k}\right)^{H} \boldsymbol{y}=\sum_{u=0}^{U-1}\left(h_{\tilde{s}_{k 1}}^{(u)}\right)^{*} \boldsymbol{c}_{k}^{H} \boldsymbol{y}_{u}
$$

Furthermore, let $\mathcal{C}_{k}$ be a set containing the $d_{x}$ indices of $\boldsymbol{c}_{k}$ having non-zero entries. Then (8) can be further simplified to

$$
\boldsymbol{h}_{\tilde{s}_{k 1}}^{H}\left(\boldsymbol{I}_{U} \otimes \boldsymbol{c}_{k}\right)^{H} \boldsymbol{y}=\sum_{u=0}^{U-1} \sum_{m \in \mathcal{C}_{k}}\left(h_{\tilde{s}_{k 1}}^{(u)}\right)^{*} c_{k m}^{*} y_{u m}
$$

\footnotetext{
${ }^{2}$ Throughout the paper, $x, \tilde{x}$ and $\hat{x}$ represent the symbol transmitted, the specific symbol being tested by the search algorithms and the final detected symbol, respectively.
} 
The second term of (7) can be simplified as

$$
\begin{aligned}
& \boldsymbol{h}_{\tilde{s}_{k 1}}^{H}\left(\boldsymbol{I}_{U} \otimes \boldsymbol{c}_{k}\right)^{H}\left(\boldsymbol{I}_{U} \otimes \boldsymbol{c}_{i}\right) \boldsymbol{h}_{\tilde{s}_{i 1}}=\boldsymbol{h}_{\tilde{s}_{k 1}}^{H}\left(\boldsymbol{I}_{U} \otimes \boldsymbol{c}_{k}^{H} \boldsymbol{c}_{i}\right) \boldsymbol{h}_{\tilde{s}_{i 1}} \\
& =\boldsymbol{h}_{\tilde{s}_{k 1}}^{H}\left(\boldsymbol{I}_{U} \otimes \sum_{m \in \mathcal{C}_{k} \cap \mathcal{C}_{i}} c_{k m}^{*} c_{i m}\right) \boldsymbol{h}_{\tilde{s}_{i 1}} \\
& =\left(\sum_{m \in \mathcal{C}_{k} \cap \mathcal{C}_{i}} c_{k m}^{*} c_{i m}\right) \boldsymbol{h}_{\tilde{s}_{k 1}}^{H} \boldsymbol{h}_{\tilde{s}_{i 1}}
\end{aligned}
$$

where $\mathcal{C}_{k} \cap \mathcal{C}_{i}$ gives a set containing the indices where both $\boldsymbol{c}_{k}$ and $\boldsymbol{c}_{i}$ have non-zero entries. Upon substituting (9) and (10) into (7), we obtain

$$
\begin{aligned}
\hat{\boldsymbol{x}}= & \arg \max _{\tilde{\boldsymbol{x}} \in \mathcal{X}^{K}}\left\{\sum_{k=1}^{K} \sum_{u=0}^{U-1} \sum_{m \in \mathcal{C}_{k}} \Re\left\{\tilde{s}_{k 2}^{*}\left(h_{\tilde{s}_{k 1}}^{(u)}\right)^{*} c_{k m}^{*} y_{u m}\right\}\right. \\
& \left.-\frac{1}{2} \sum_{k=1}^{K} \sum_{i=1}^{K}\left(\sum_{m \in \mathcal{C}_{k} \cap \mathcal{C}_{i}} c_{k m}^{*} c_{i m}\right) \tilde{s}_{k 2}^{*} \boldsymbol{h}_{\tilde{s}_{k 1}}^{H} \boldsymbol{h}_{\tilde{s}_{i 1}} \tilde{s}_{i 2}\right\}
\end{aligned}
$$

From (11) we know that although $\mathcal{C}_{k}$ has only $d_{x}$ non-zero elements, which can be exploited for significantly reducing the computation, the MLD's complexity is still $\mathcal{O}\left(M^{K}\right)$. Therefore, the MLD described by (11) is impractical, especially, when the SM-SCDMA systems are proposed for supporting numerous users (devices). Next, we consider the symbol-based MAPD, which facilitates the employment of the MPA, as shown in Section III-C.

\section{B. Maximum A-Posteriori Detection (MAPD)}

Given the observation of $y$ in (4), the symbol-by-symbol based MAPD detects the $l$ th user's symbol via maximizing the posteriori probability as

$$
\begin{aligned}
\hat{x}_{l} & =\arg \max _{\tilde{x}_{l} \in \mathcal{X}}\left\{P\left(\tilde{x}_{l} \mid \boldsymbol{y}\right)\right\}=\arg \max _{\tilde{x}_{l} \in \mathcal{X}}\left\{P\left(\tilde{x}_{l}\right) p\left(\boldsymbol{y} \mid \tilde{x}_{l}\right)\right\} \\
& =\arg \max _{\tilde{x}_{l} \in \mathcal{X}}\left\{\sum_{\boldsymbol{x}_{l} \in \mathcal{X}^{K-1}} P\left(\boldsymbol{x}_{l}, \tilde{x}_{l}\right) p\left(\boldsymbol{y} \mid \boldsymbol{x}_{l}, \tilde{x}_{l}\right)\right\}
\end{aligned}
$$

where $p(x \mid a)$ is the conditional probability density function (PDF) of $x$ given $a, P(a)$ is the a-priori probability of $a$, while $x_{l}$ is a $(K-1)$-element vector obtained from $x$ by removing the $l$ th user's symbol. Since the observations $\left\{y_{u n}\right\}$ are independent for given $\left(\boldsymbol{x}_{l}, \tilde{x}_{l}\right),(12)$ can be written in detail as

$$
\hat{x}_{l}=\arg \max _{\tilde{x}_{l} \in \mathcal{X}}\left\{\sum_{\boldsymbol{x}_{l} \in \mathcal{X}^{K-1}} P\left(\boldsymbol{x}_{l}, \tilde{x}_{l}\right) \prod_{u=0}^{U-1} \prod_{n=0}^{N-1} p\left(y_{u n} \mid \boldsymbol{x}_{l}, \tilde{x}_{l}\right)\right\}
$$

Let $\mathcal{K}_{l}$ be a set containing all the interfering users sharing at least one (non-zero) chip with user $l$. Let furthermore $x_{[n]}$ be a $d_{f}$-length vector containing the symbols sent by the users sharing the $n$th chip of the spreading sequences. Then (13) can be further simplified to

$$
\hat{x}_{l}=\arg \max _{\tilde{x}_{l} \in \mathcal{X}}\left\{\sum_{\boldsymbol{x}_{l}^{\prime} \in \mathcal{X}^{\left|\mathcal{K}_{l}\right|}} P\left(\boldsymbol{x}_{l}^{\prime}, \tilde{x}_{l}\right) \prod_{u=0}^{U-1} \prod_{n \in \mathcal{C}_{l}} p\left(y_{u n} \mid \boldsymbol{x}_{[n]}\right)\right\}
$$

where $\left|\mathcal{K}_{l}\right|$ is the cardinality of $\mathcal{K}_{l}$, and $x_{l}^{\prime}$ is of $\left|\mathcal{K}_{l}\right|$-length. In (14), when $x_{[n]}$ is given, the PDF of $p\left(y_{u n} \mid x_{[n]}\right)$ can be expressed as

$$
p\left(y_{u n} \mid \boldsymbol{x}_{[n]}\right)=\frac{1}{2 \pi \sigma^{2}} \exp \left(-\frac{\left\|y_{u n}-\sum_{k \in \mathcal{D}_{n}} h_{s_{k 1}}^{(u)} s_{k 2} c_{k n}\right\|^{2}}{2 \sigma^{2}}\right)
$$

where we define $\mathcal{D}_{n}$ the set containing the indices of the users conveying information using the $n$th chip of the spreading codes.

Equation (14) shows that the complexity of detecting user $l$ is $\mathcal{O}\left(M^{\left|\mathcal{K}_{l}\right|}\right)$. When regular sparse codes are employed, we have $\left|\mathcal{K}_{l}\right|=d_{x}\left(d_{f}-1\right)$, which is a constant usually much smaller than $K$. Hence, employing sparse codes for spreading is capable of reducing the detection complexity of MAPD. Furthermore, when the MAPD is implemented by an approximated algorithm, the detection complexity can be further reduced [2].

\section{Message Passing Algorithm Aided Detection (MPAD)}

Similar to the classic low-density parity-check codes [50], the input-output relationship in the proposed SM-SCDMA system can be described by a factor graph [2,24], as shown in Fig. 2 for the parameters specified in the caption. In this factor graph, the $K$ symbols of the form $x_{k}=s_{k 1} \mid s_{k 2}$ and sent by $K$ users are represented by $K$ variable nodes, while the $U N$ observations in $\boldsymbol{y}$ act as $N$ function (or check) nodes, with each having $U$ channel inputs provided by the $U$ receive antennas. As shown in the figure, users 1,6 and 7 share the 0th function node, which has two observations obtained from the first and second receive antennas respectively. Hence, while these three users do interfere with each other on the 0th chip, information carried by the different chips activated by the three users can also be transferred from one chip to another via the 0th function node. By doing this, the overall detection performance of SM-SCDMA may be significantly improved. Below we detail the MPAD.

Let us define two sets as

$$
\begin{aligned}
\overline{\mathcal{K}}_{j} & =\left\{i: 0 \leq i \leq N-1, c_{j i} \neq 0\right\}, j=1,2, \ldots, K \\
\overline{\mathcal{C}}_{i} & =\left\{j: 1 \leq j \leq K, c_{j i} \neq 0\right\}, i=0,1, \ldots, N-1
\end{aligned}
$$

where we have $\left|\overline{\mathcal{K}}_{j}\right|=d_{x}$, representing the $d_{x}$ connections with the variable node $j$, and $\left|\overline{\mathcal{C}}_{i}\right|=d_{f}$, giving the $d_{f}$ connections with the function node $i$.

Let us explicitly express the symbol set as $\mathcal{X}=$ $\left\{a_{0}, a_{1}, \ldots, a_{M-1}\right\}$, where $M=M_{1} M_{2}$. Let the probability $\eta_{j, i}^{a_{m},(n)}$ be a message sent from the variable node $x_{j}$ to the function node $f_{i}$ (corresponding to the $i$ th chip of the spreading sequences) during the $n$th iteration. Note that here $\eta_{j, i}^{a_{m},(n)}$ is the probability that we have $x_{j}=a_{m}$, given all the messages received by $x_{j}$ from all of its neighboring function nodes, excluding $f_{i}$. Similarly, let the probability $\delta_{i, j}^{a_{m},(n)}$ represent a message sent from the function node $i$ to the variable node $j$ during the $n$th iteration. Explicitly, this is the probability that $x_{j}=a_{m}$, given the specific messages received by $f_{i}$ from all its neighboring variable nodes, excluding $x_{j}$. 


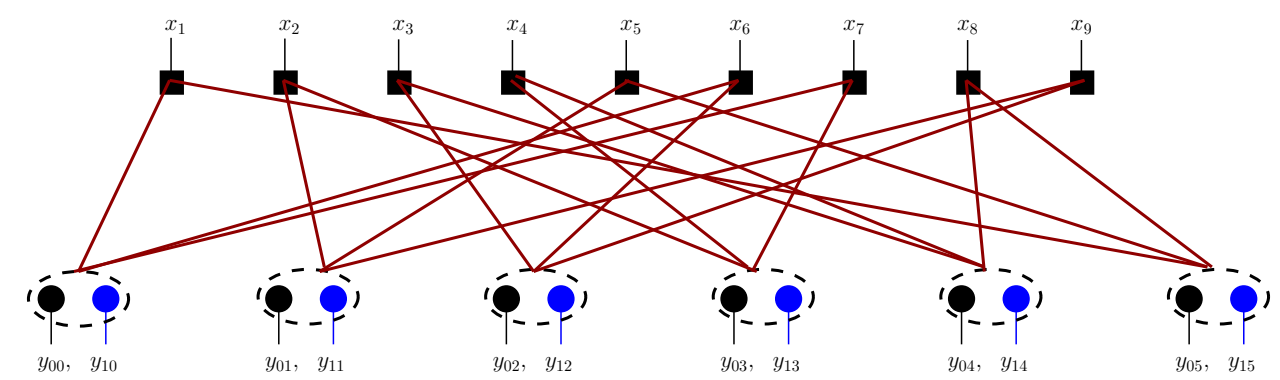

Fig. 2. Factor graph representation of the SM-SCDMA system with the parameters of $U=2, N=6, K=9$, and $d_{x}=2, d_{f}=3$.

Then, following $[51,52]$ and with the aid of Fig. 2, the MPAD can be described as follows.

Initially, we set $\eta_{j, i}^{a_{m},(0)}=1 / M$ for all $a_{m} \in \mathcal{X}$ and for any specific $(j, i)$ pairs, where $j \in \overline{\mathcal{K}}_{j}$ and $i \in \overline{\mathcal{C}}_{i}$. Then, during the $n$ th, $n \geq 0$, iteration, the probability $\delta_{i, j}^{a_{m},(n)}$ can be computed as

$$
\begin{aligned}
\delta_{i, j}^{a_{m},(n)}= & \sum_{\boldsymbol{x}_{[i]} \in \mathcal{X}^{d_{f}-1}, x_{j}=a_{m}}\left(\prod_{x_{v} \in \boldsymbol{x}_{[i]} \backslash x_{j}} \eta_{j, i}^{x_{v},(n)}\right) \\
\times & \prod_{u=0}^{U-1} p\left(y_{u i} \mid \boldsymbol{x}_{[i]}, x_{j}=a_{m}\right), \\
& m=0, \ldots, M-1
\end{aligned}
$$

for $i \in \overline{\mathcal{C}}_{i}$ and $j \in \overline{\mathcal{K}}_{j}$, where $\prod_{x_{v} \in \boldsymbol{x}_{[i]} \backslash x_{j}} \eta_{j, i}^{x_{v},(n)}$ is the a-priori probability of a given $x_{[i]}$ with $x_{j}=a_{m}$, while $p\left(y_{u i} \mid \boldsymbol{x}_{[i]}, x_{j}=a_{m}\right)$ is given by (15).

Then, during the $(n+1)$ st iteration, the values $\delta_{i, j}^{a_{m}},(n)$ for $i \in \overline{\mathcal{C}}_{i}$ and $j \in \overline{\mathcal{K}}_{j}$ are used to compute $\eta_{j, i}^{a_{m},(n+1)}$ for $j \in \overline{\mathcal{K}}_{j}$ and $i \in \overline{\mathcal{C}}_{i}$, using the formula of

$$
\eta_{j, i}^{a_{m},(n+1)}=\xi_{j, i} \prod_{v \in \overline{\mathcal{K}}_{j} \backslash i} \delta_{v, j}^{a_{m},(n)}, m=0,1, \ldots, M-1
$$

where $\xi_{j, i}$ is applied to make $\sum_{m=0}^{M-1} \eta_{j, i}^{a_{m},(n+1)}=1$.

Finally, when the affordable number of iterations is exhausted, the data symbols sent from the $K$ users are detected as

$$
x_{j}=\arg \max _{a_{m}} \prod_{v \in \overline{\mathcal{K}}_{j}} \delta_{v, j}^{a_{m},(n)}, k=1,2, \ldots, K
$$

The complexity of the MPAD is dependent on the number of users sharing a chip. When regular sparse codes are used, the complexity per user can be shown to be $\mathcal{O}\left(M^{d_{f}}\right)$ [52]. Hence, provided that $d_{x}, d_{f} \geq 2$, the MPAD has a lower complexity than the MAPD considered in Section III-B, which has the complexity of $\mathcal{O}\left(M^{d_{x}\left(d_{f}-1\right)}\right)$ per user for regular sparse codes.

\section{Performance Analysis}

This section contributes to the error performance analysis of SM-SCDMA systems. We first analyze the single-user BER performance, which is the lower-bound BER of SMSCDMA systems. Then, based on the single-user BER, we derive formulas for approximating the BER of SM-SCDMA systems supporting $K$ users.

\section{A. Analysis of Single-User Average BER}

When the SM-SCDMA system supports only a single user, the MLD of (7) becomes

$$
\hat{x}=\arg \max _{\tilde{x} \in \mathcal{X}}\left\{\Re\left\{\tilde{s}_{2}^{*} \boldsymbol{h}_{\tilde{s}_{1}}^{H}\left(\boldsymbol{I}_{U} \otimes \boldsymbol{c}_{k}\right)^{H} \boldsymbol{y}\right\}-\frac{1}{2}\left\|\boldsymbol{h}_{\tilde{s}_{1}} \tilde{s}_{2}\right\|^{2}\right\}
$$

where the index $k$ (except $\boldsymbol{c}_{k}$ ) is dropped for notational convenience. A detection error occurs, when $\hat{x} \neq x$, which resulted from the event that there is at least one $\tilde{x} \neq x$, yielding

$$
\begin{aligned}
\Re\left\{\tilde{s}_{2}^{*} \boldsymbol{h}_{\tilde{s}_{1}}^{H}\left(\boldsymbol{I}_{U} \otimes \boldsymbol{c}_{k}\right)^{H} \boldsymbol{y}\right\}-\frac{1}{2}\left\|\boldsymbol{h}_{\tilde{s}_{1}} \tilde{s}_{2}\right\|^{2} \\
\quad>\Re\left\{s_{2}^{*} \boldsymbol{h}_{s_{1}}^{H}\left(\boldsymbol{I}_{U} \otimes \boldsymbol{c}_{k}\right)^{H} \boldsymbol{y}\right\}-\frac{1}{2}\left\|\boldsymbol{h}_{s_{1}} s_{2}\right\|^{2}
\end{aligned}
$$

Let us arrange (21) as

$$
\begin{array}{r}
\Re\left\{\left(\boldsymbol{h}_{\tilde{s}_{1}} \tilde{s}_{2}-\boldsymbol{h}_{s_{1}} s_{2}\right)^{H}\left(\boldsymbol{I}_{U} \otimes \boldsymbol{c}_{k}\right)^{H} \boldsymbol{y}\right\} \\
>\frac{1}{2}\left(\left\|\boldsymbol{h}_{\tilde{s}_{1}} \tilde{s}_{2}\right\|^{2}-\left\|\boldsymbol{h}_{s_{1}} s_{2}\right\|^{2}\right)
\end{array}
$$

Then, upon substituting $y$ from (4) for $K=1$ into (22), we obtain

$$
\Re\left\{\left(\boldsymbol{h}_{\tilde{s}_{1}} \tilde{s}_{2}-\boldsymbol{h}_{s_{1}} s_{2} \|\right)^{H} \boldsymbol{n}^{\prime}\right\}>\frac{1}{2}\left(\left\|\boldsymbol{h}_{\tilde{s}_{1}} \tilde{s}_{2}-\boldsymbol{h}_{s_{1}} s_{2}\right\|^{2}\right)
$$

where $\boldsymbol{n}^{\prime}=\left(\boldsymbol{I}_{U} \otimes \boldsymbol{c}\right)^{H} \boldsymbol{n}$, which obeys the PDF of $\mathcal{C N}\left(0,2 \sigma^{2} \boldsymbol{I}_{U}\right)$. Hence, the probability that the transmitted symbol $x$ is detected as $\tilde{x}$, which is usually referred to as the PEP, is given by

$$
\begin{aligned}
P_{E P}(x \rightarrow \tilde{x}) & =E_{\boldsymbol{H}}\left[Q\left(\sqrt{\frac{1}{4 \sigma^{2}}\left\|\boldsymbol{h}_{\tilde{s}_{1}} \tilde{s}_{2}-\boldsymbol{h}_{s_{1}} s_{2}\right\|^{2}}\right)\right] \\
& =E_{\boldsymbol{H}}\left[Q\left(\sqrt{\frac{\gamma_{0}}{2}\left\|\boldsymbol{h}_{\tilde{s}_{1}} \tilde{s}_{2}-\boldsymbol{h}_{s_{1}} s_{2}\right\|^{2}}\right)\right]
\end{aligned}
$$

where $Q(x)$ is the Gaussian $Q$-function defined as $Q(x)=$ $(2 \pi)^{-1 / 2} \int_{x}^{\infty} e^{-t^{2} / 2} d t$, and $E_{\boldsymbol{H}}[\cdot]$ denotes the expectation with respect to the MIMO channel $\boldsymbol{H}$ between the user and the BS.

As shown in $[36,53]$, the average bit error rate (ABER) of SM schemes may be approximately evaluated from the unionbound expressed as

$$
\begin{aligned}
\bar{P}_{b S} \leq & \frac{1}{M_{1} M_{2} b} \sum_{m_{1}=0}^{M_{1}-1} \sum_{m_{2}=0}^{M_{2}-1} \sum_{\tilde{m}_{1}=0}^{M_{1}-1} \sum_{\tilde{m}_{2}=0}^{M_{2}-1} \\
& \times D\left(\boldsymbol{b}_{1}^{\left(m_{1}\right)}\left|\boldsymbol{b}_{2}^{\left(m_{2}\right)}, \tilde{\boldsymbol{b}}_{1}^{\left(\tilde{m}_{1}\right)}\right| \tilde{\boldsymbol{b}}_{2}^{\left(\tilde{m}_{2}\right)}\right) \\
& \times P_{E P}\left(s_{1}^{\left(m_{1}\right)}\left|s_{2}^{\left(m_{2}\right)} \rightarrow \tilde{s}_{1}^{\left(\tilde{m}_{1}\right)}\right| \tilde{s}_{2}^{\left(\tilde{m}_{2}\right)}\right)
\end{aligned}
$$


where $D(\cdot, \cdot)$ is the Hamming distance between the two binary entries, and $\boldsymbol{b}_{i}^{(m)}, s_{i}^{(m)}$ represent the $m$ th realizations in bits and symbols. Furthermore, in [53], the closed-form formulas for the PEP of (24) have been derived in the context of different fading channels. Specifically, in this paper, we are only interested in the iid Rayleigh fading channels. In this case, following the analysis in [53], $P_{E P}(x \rightarrow \tilde{x})$ can be analyzed by dividing it into the following three cases:

1) $\left(M_{1}\right.$ SSK-Error-Only): $P_{E P}(x \rightarrow \tilde{x})=P_{E P}\left(s_{1} \mid s_{2} \rightarrow\right.$ $\left.\tilde{s}_{1} \mid s_{2}\right)$

2) ( $M_{2}$ APM-Error-Only): $P_{E P}(x \rightarrow \tilde{x})=P_{E P}\left(s_{1} \mid s_{2} \rightarrow\right.$ $\left.s_{1} \mid \tilde{s}_{2}\right)$

3) (Both $M_{1} \mathrm{SSK}$ and $M_{2} \mathrm{APM}$ in Error): $P_{E P}(x \rightarrow \tilde{x})=$ $P_{E P}\left(s_{1}\left|s_{2} \rightarrow \tilde{s}_{1}\right| \tilde{s}_{2}\right)$.

Upon invoking the alternative representation for the $Q$ function $[54]^{3}$, these probabilities can be explicitly expressed as

$$
\begin{aligned}
P_{E P}\left(s_{1}\left|s_{2} \rightarrow \tilde{s}_{1}\right| s_{2}\right)=\frac{1}{\pi} \int_{0}^{\pi / 2}\left(\frac{2 \sin ^{2} \theta}{2 \sin ^{2} \theta+\gamma_{0}\left|s_{2}\right|^{2}}\right)^{U} d \theta \\
P_{E P}\left(s_{1}\left|s_{2} \rightarrow s_{1}\right| \tilde{s}_{2}\right)=\frac{1}{\pi} \\
\quad \times \int_{0}^{\pi / 2}\left(\frac{4 \sin ^{2} \theta}{4 \sin ^{2} \theta+\gamma_{0}\left|\tilde{s}_{2}-s_{2}\right|^{2}}\right)^{U} d \theta \\
P_{E P}\left(s_{1}\left|s_{2} \rightarrow \tilde{s}_{1}\right| \tilde{s}_{2}\right)=\frac{1}{\pi} \\
\quad \times \int_{0}^{\pi / 2}\left(\frac{4 \sin ^{2} \theta}{4 \sin ^{2} \theta+\gamma_{0}\left(\left|\tilde{s}_{2}\right|^{2}+\left|s_{2}\right|^{2}\right)}\right)^{U} d \theta
\end{aligned}
$$

Equations (26a)-(26c) show that the fading channel effects have been averaged out in the PEP. This means that the computation of (25) does not need to consider the specific $M_{1}$ SSK symbols, only the $M_{2} \mathrm{APM}$ symbols need to be considered. Therefore, (25) can be simplified to [53]

$$
\begin{aligned}
\bar{P}_{b S} \leq & \frac{M_{1} b_{1}}{2 M_{2} b} \sum_{m_{2}=0}^{M_{2}-1} P_{E P}\left(s_{1}\left|s_{2}^{\left(m_{2}\right)} \rightarrow \tilde{s}_{1}\right| s_{2}^{\left(m_{2}\right)}\right) \\
+ & \frac{1}{M_{2} b} \sum_{m_{2}=0}^{M_{2}-1} \sum_{\tilde{m}_{2} \neq m_{2}}^{M_{2}-1} D\left(\boldsymbol{b}_{2}^{\left(m_{2}\right)}, \tilde{\boldsymbol{b}}_{2}^{\left(\tilde{m}_{2}\right)}\right) \\
& \times P_{E P}\left(s_{1}\left|s_{2}^{\left(m_{2}\right)} \rightarrow s_{1}\right| \tilde{s}_{2}^{\left(\tilde{m}_{2}\right)}\right) \\
+ & \frac{1}{M_{2} b} \sum_{m_{2}=0}^{M_{2}-1} \sum_{\tilde{m}_{2} \neq m_{2}}^{M_{2}-1}\left[\frac{b_{1} M_{1}}{2}+\left(M_{1}-1\right)\right. \\
& \left.\times D\left(\boldsymbol{b}_{2}^{\left(m_{2}\right)}, \tilde{\boldsymbol{b}}_{2}^{\left(\tilde{m}_{2}\right)}\right)\right] P_{E P}\left(s_{1}^{\left(m_{1}\right)}\left|s_{2}^{\left(m_{2}\right)} \rightarrow \tilde{s}_{1}^{\left(\tilde{m}_{1}\right)}\right| \tilde{s}_{2}^{\left(\tilde{m}_{2}\right)}\right)
\end{aligned}
$$

where the first and third terms at the righthand side are obtained by exploiting the fact that the average number of erroneous bits between a pair of $\left(\boldsymbol{b}_{1}, \tilde{b}_{1}\right)$ is $b_{1} M_{1} /\left[2\left(M_{1}-1\right)\right]$. Furthermore, in $P_{E P}(\cdot), s_{i} \rightarrow \tilde{s}_{i}$ or $s_{i}^{(m)} \rightarrow \tilde{s}_{i}^{(\tilde{m})}$ means that the error event corrupts the transmitted $s_{i}$ (or $s_{i}^{(m)}$ ) into $\tilde{s}_{i}$ (or $\left.\tilde{s}_{i}^{(\tilde{m})}\right)$.

$$
{ }^{3} Q(x)=\pi^{-1} \int_{0}^{\pi / 2} \exp \left(-\frac{x^{2}}{2 \sin ^{2} \theta}\right) d \theta
$$

Note first that if we assume that the $M_{2}$ APM uses Gray coding [54], the ABER of the $M_{2}$ APM is given by

$$
\begin{aligned}
\bar{P}_{b}\left(M_{2} \mathrm{APM}\right)= & \frac{1}{M_{2} b_{1}} \sum_{m_{2}=0}^{M_{2}-1} \sum_{\tilde{m}_{2} \neq m_{2}}^{M_{2}-1} D\left(\boldsymbol{b}_{2}^{\left(m_{2}\right)}, \tilde{\boldsymbol{b}}_{2}^{\left(\tilde{m}_{2}\right)}\right) \\
& \times P_{E P}\left(s_{1}\left|s_{2}^{\left(m_{2}\right)} \rightarrow s_{1}\right| \tilde{s}_{2}^{\left(\tilde{m}_{2}\right)}\right) \\
= & \bar{P}_{s}\left(M_{2} \mathrm{APM}\right) / b_{1}
\end{aligned}
$$

where $\bar{P}_{s}\left(M_{2} \mathrm{APM}\right)$ represents the average symbol error probability of $M_{2}$ APM. For different types of APMs, there are closed-form formulas to evaluate their ABER and average symbol error probability, which can be found in references, such as, [54-56]. Hence, we can use the existing formulas for $\bar{P}_{b}\left(M_{2} \mathrm{APM}\right)$ as well as $\bar{P}_{s}\left(M_{2} \mathrm{APM}\right)$, and evaluate $(27 \mathrm{~b})$ as $b_{1} \bar{P}_{b}\left(M_{2} \mathrm{APM}\right) / b$ or $\bar{P}_{s}\left(M_{2} \mathrm{APM}\right) / b$.

Note secondly that, since $U$ in (26) is an integer, according to (64) of [57] or (5A.4b) of [54], the PEPs in (26a) - (26c) can be expressed in closed form as

$$
P_{E P}(x \rightarrow \tilde{x})=\left(\frac{1-\mu}{2}\right)^{U} \sum_{u=0}^{U-1}\left(\begin{array}{l}
U \\
u
\end{array}\right)\left(\frac{1+\mu}{2}\right)^{u}
$$

where $\mu$ corresponding to (26a), (26b) and (26c) is respectively defined as

$$
\mu= \begin{cases}\sqrt{\frac{\gamma_{0}\left|s_{2}\right|^{2}}{\gamma_{0}\left|s_{2}\right|^{2}+2},} & \text { if (26a) for Case (a) } \\ \sqrt{\frac{\gamma_{0}\left|\tilde{s}_{2}-s_{2}\right|^{2}}{\gamma_{0}\left|\tilde{s}_{2}-s_{2}\right|^{2}+4},} & \text { if (26b) for Case (b) } \\ \sqrt{\frac{\gamma_{0}\left(\left|\tilde{s}_{2}\right|^{2}+\left|s_{2}\right|^{2}\right)}{\gamma_{0}\left(\left|\tilde{s}_{2}\right|^{2}+\left|s_{2}\right|^{2}\right)+4},} & \text { if (26c) for Case (c) }\end{cases}
$$

The PEPs can be readily computed with the aid of (29).

Equation (27) gives a bound for the ABER or the approximate ABER (if the SNR is sufficiently high) of the single-user SM-SCDMA system, without informing us whether the error is dominated by the $M_{1}$ SSK or the $M_{2}$ APM. This information is sometimes important in design. For example, if a designer knows that one is more reliable than the other one, the two modulation schemes can be used for unequal protection of multimedia information. Another consideration in the design is that the appropriate $M_{1} \mathrm{SSK}$ and $M_{2} \mathrm{APM}$ may be chosen so that both the modulation schemes give a similar ABER. This is because the overall ABER is always dominated by the less reliable one. In this case, the reliability of the more reliable one may be slightly reduced by transmitting at a higher rate, without unduly increasing the overall ABER.

Similar to the derivation of (25) [54], the ABER of the $M_{1}$ SSK and that of the $M_{2}$ APM have the following union- 
bounds:

$$
\begin{aligned}
& \bar{P}_{b S}\left(M_{1} \mathrm{SSK}\right) \leq \frac{1}{M_{1} b_{1}} \sum_{m_{1}=0}^{M_{1}-1} \sum_{m_{2}=0}^{M_{2}-1} \sum_{\tilde{m}_{1}=0}^{M_{1}-1} \sum_{\tilde{m}_{2}=0}^{M_{2}-1} \\
& \times D\left(\boldsymbol{b}_{1}^{\left(m_{1}\right)}, \tilde{\boldsymbol{b}}_{1}^{\left(\tilde{m}_{1}\right)}\right) P_{E P}\left(s_{1}^{\left(m_{1}\right)}\left|s_{2}^{\left(m_{2}\right)} \rightarrow \tilde{s}_{1}^{\left(\tilde{m}_{1}\right)}\right| \tilde{s}_{2}^{\left(\tilde{m}_{2}\right)}\right) \\
& \bar{P}_{b S}\left(M_{2} \mathrm{APM}\right) \leq \frac{1}{M_{2} b_{2}} \sum_{m_{1}=0}^{M_{1}-1} \sum_{m_{2}=0}^{M_{2}-1} \sum_{\tilde{m}_{1}=0}^{M_{1}-1} \sum_{\tilde{m}_{2}=0}^{M_{2}-1} \\
& \times D\left(\boldsymbol{b}_{2}^{\left(m_{2}\right)}, \tilde{\boldsymbol{b}}_{2}^{\left(\tilde{m}_{2}\right)}\right) P_{E P}\left(s_{1}^{\left(m_{1}\right)}\left|s_{2}^{\left(m_{2}\right)} \rightarrow \tilde{s}_{1}^{\left(\tilde{m}_{1}\right)}\right| \tilde{s}_{2}^{\left(\tilde{m}_{2}\right)}\right)
\end{aligned}
$$

Again, considering that the PEPs shown in (26a) - (26c) for the three cases are not dependent on the fading channels, (31) and (32) can be simplified to the formulas of

$$
\begin{aligned}
& \bar{P}_{b S}(\left.M_{1} \mathrm{SSK}\right) \leq \frac{M_{1}}{2} \sum_{m_{2}=0}^{M_{2}-1} P_{E P}\left(s_{1}\left|s_{2}^{\left(m_{2}\right)} \rightarrow \tilde{s}_{1}\right| s_{2}^{\left(m_{2}\right)}\right) \\
&+ \frac{M_{1}}{2} \sum_{m_{2}=0}^{M_{2}-1} \sum_{\tilde{m}_{2} \neq m_{2}}^{M_{2}-1} P_{E P}\left(s_{1}\left|s_{2}^{\left(m_{2}\right)} \rightarrow \tilde{s}_{1}\right| \tilde{s}_{2}^{\left(\tilde{m}_{2}\right)}\right) \\
& \bar{P}_{b S}\left(M_{2} \mathrm{APM}\right) \leq \frac{M_{1}}{M_{2} b_{2}} \sum_{m_{2}=0}^{M_{2}-1} \sum_{\tilde{m}_{2} \neq m_{2}}^{M_{2}-1} D\left(\boldsymbol{b}_{2}^{\left(m_{2}\right)}, \tilde{\boldsymbol{b}}_{2}^{\left(\tilde{m}_{2}\right)}\right) \\
& \quad \times P_{E P}\left(s_{1}\left|s_{2}^{\left(m_{2}\right)} \rightarrow s_{1}\right| \tilde{s}_{2}^{\left(\tilde{m}_{2}\right)}\right) \\
&+\frac{M_{1}\left(M_{1}-1\right)}{M_{2} b_{2}} \sum_{m_{2}=0}^{M_{2}-1} \sum_{\tilde{m}_{2} \neq m_{2}-1}^{M_{2}} D\left(\boldsymbol{b}_{2}^{\left(m_{1}\right)}, \tilde{\boldsymbol{b}}_{2}^{\left(\tilde{m}_{1}\right)}\right) \\
& \quad \times P_{E P}\left(s_{1}\left|s_{2}^{\left(m_{2}\right)} \rightarrow \tilde{s}_{1}\right| \tilde{s}_{2}^{\left(\tilde{m}_{2}\right)}\right)
\end{aligned}
$$

In (33) and (34), the PEPs are respectively given in (26a) (26c), which can be computed using (29) associated with (30).

\section{B. Analysis of Approximate BER of SM-SCDMA Systems}

In this section, we analyze the ABER of SM-SCDMA systems with MLD. In practice we are usually interested in the performance of an uncoded system at the specific ABER of about $10^{-3}$, which can be readily corrected by FEC codes. For this ABER or a lower ABER, we can realize that the probability of two or more users being simultaneously in error at the same transmission instant should be negligible, provided that the number of (active) users is significantly smaller than $1 /$ ABER. Hence, in order to make the analysis tractable, we assume that there is at most one user among the $(K-1)$ interfering users that may be in error.

Before we start analyzing the ABER of SM-SCDMA systems, we can readily obtain from (25) and (26a) - (26c) that the ASER of the single-user SM-SCDMA system is bounded as

$$
\begin{aligned}
& \bar{P}_{s S} \leq \frac{1}{M_{1} M_{2}} \sum_{m_{1}=0}^{M_{1}-1} \sum_{m_{2}=0}^{M_{2}-1} \sum_{\tilde{m}_{1}=0}^{M_{1}-1} \sum_{\tilde{m}_{2}=0}^{M_{2}-1} \\
& \times P_{E P}\left(s_{1}^{\left(m_{1}\right)}\left|s_{2}^{\left(m_{2}\right)} \rightarrow \tilde{s}_{1}^{\left(\tilde{m}_{1}\right)}\right| \tilde{s}_{2}^{\left(\tilde{m}_{2}\right)}\right) \\
& =\frac{M_{1}-1}{M_{2}} \sum_{m_{2}=0}^{M_{2}-1} P_{E P}\left(s_{1}\left|s_{2}^{\left(m_{2}\right)} \rightarrow \tilde{s}_{1}\right| s_{2}^{\left(m_{2}\right)}\right) \\
& +\frac{M_{1}-1}{M_{2}} \sum_{m_{2}=0}^{M_{2}-1} \sum_{\tilde{m}_{2} \neq m_{2}}^{M_{2}-1} P_{E P}\left(s_{1}\left|s_{2}^{\left(m_{2}\right)} \rightarrow s_{1}\right| \tilde{s}_{2}^{\left(\tilde{m}_{2}\right)}\right) \\
& +\frac{M_{1}-1}{M_{2}} \sum_{m_{2}=0}^{M_{2}-1} \sum_{\tilde{m}_{2} \neq m_{2}}^{M_{2}-1} P_{E P}\left(s_{1}\left|s_{2}^{\left(m_{2}\right)} \rightarrow \tilde{s}_{1}\right| \tilde{s}_{2}^{\left(\tilde{m}_{2}\right)}\right)
\end{aligned}
$$

This probability will be used later for computing the ABER of the SM-SCDMA systems.

Let us now assume that there is a desired user indexed in the same way as in Section IV-A. Then, provided that all the $(K-1)$ interfering users are correctly detected, the ABER of the reference user is the same as (27).

By contrast, when an interfering user indexed as ' $k$ ', which can be any of the $(K-1)$ interfering users, is in error, we have the pairwise erroneous event of

$$
\begin{aligned}
& \left\|\boldsymbol{y}-\left(\boldsymbol{I}_{U} \otimes \boldsymbol{c}\right) \boldsymbol{h}_{\tilde{s}_{1}} \tilde{s}_{2}-\left(\boldsymbol{I}_{U} \otimes \boldsymbol{c}_{k}\right) \boldsymbol{h}_{\tilde{s}_{k 1}} \tilde{s}_{k 2}\right\|^{2} \\
& \quad<\left\|\boldsymbol{y}-\left(\boldsymbol{I}_{U} \otimes \boldsymbol{c}\right) \boldsymbol{h}_{s_{1}} s_{2}-\left(\boldsymbol{I}_{U} \otimes \boldsymbol{c}_{k}\right) \boldsymbol{h}_{s_{k 1}} s_{k 2}\right\|^{2}
\end{aligned}
$$

from which we can derive the PEP, given as

$$
P_{E P}^{(i)}\left(x, x_{k} \rightarrow \tilde{x}, \tilde{x}_{k}\right)=E_{\boldsymbol{H}}\left[Q\left(\sqrt{2 \sum_{u=0}^{U-1} \gamma_{u}^{(i)}}\right)\right]
$$

where $x, \tilde{x}, x_{k}$ and $\tilde{x}_{k}$ represent respectively $s_{1}\left|s_{2}, \tilde{s}_{1}\right| \tilde{s}_{2}$, $s_{k 1} \mid s_{k 2}$ and $\tilde{s}_{k 1} \mid \tilde{s}_{k 2}$, while

$$
\begin{aligned}
\gamma_{u}^{(i)}= & \gamma_{0}\left\|\boldsymbol{c} h_{\tilde{s}_{1}}^{(u)} \tilde{s}_{2}+\boldsymbol{c}_{k} h_{\tilde{s}_{k 1}}^{(u)} \tilde{s}_{k 2}-\boldsymbol{c} h_{s_{1}}^{(u)} s_{2}-\boldsymbol{c}_{k} h_{s_{k 1}}^{(u)} s_{k 2}\right\|^{2} / 4 \\
& =\gamma_{0} \alpha / 4
\end{aligned}
$$

where a superscript $i$ is added to distinguish the different cases to be considered later.

When expressing the realizations of $x, \tilde{x}, x_{k}, \tilde{x}_{k}$ as $s_{1}^{\left(m_{1}\right)}\left|s_{2}^{\left(m_{2}\right)}, \tilde{s}_{1}^{\left(m_{1}\right)}\right| \tilde{s}_{2}^{\left(m_{2}\right)}, s_{k 1}^{\left(m_{k 1}\right)}\left|s_{k 2}^{\left(m_{k 2}\right)}, \tilde{s}_{k 1}^{\left(m_{k 1}\right)}\right| \tilde{s}_{k 2}^{\left(m_{k 2}\right)}$, similar to (25), we can represent the ABER of the SM-SCDMA system under the condition of a given interfering user $k$ being incorrectly detected as

$$
\begin{aligned}
\bar{P}_{b M}(k) \approx & \frac{1}{M_{1} M_{2} b} \sum_{m_{1}=0}^{M_{1}-1} \sum_{m_{2}=0}^{M_{2}-1} \sum_{\tilde{m}_{1}=0}^{M_{1}-1} \sum_{\tilde{m}_{2}=0}^{M_{2}-1} \sum_{m_{k 1}=0}^{M_{1}-1} \sum_{m_{k 2}=0}^{M_{2}-1} \sum_{\tilde{m}_{k 1}=0}^{M_{1}-1} \\
& \times \sum_{\tilde{m}_{k 2}=0}^{M_{2}-1} D\left(\boldsymbol{b}_{1}^{\left(m_{1}\right)}\left|\boldsymbol{b}_{2}^{\left(m_{2}\right)}, \tilde{\boldsymbol{b}}_{1}^{\left(\tilde{m}_{1}\right)}\right| \tilde{\boldsymbol{b}}_{2}^{\left(\tilde{m}_{2}\right)}\right) \\
& \times P_{E P}\left(s_{1}^{\left(m_{1}\right)}\left|s_{2}^{\left(m_{2}\right)}, s_{k 1}^{\left(m_{k 1}\right)}\right| s_{k 2}^{\left(m_{k 2}\right)}\right. \\
& \left.\rightarrow \tilde{s}_{1}^{\left(\tilde{m}_{1}\right)}\left|\tilde{s}_{2}^{\left(\tilde{m}_{2}\right)}, \tilde{s}_{k 1}^{\left(\tilde{m}_{k 1}\right)}\right| \tilde{s}_{k 2}^{\left(\tilde{m}_{k 2}\right)}\right)
\end{aligned}
$$

where we simply use ' $\approx$ ' to replace ' $<$ ', since certain assumptions resulting in approximation are invoked, as noted at the 
beginning of this section. In order to evaluate (39), we have to consider the following nine cases:

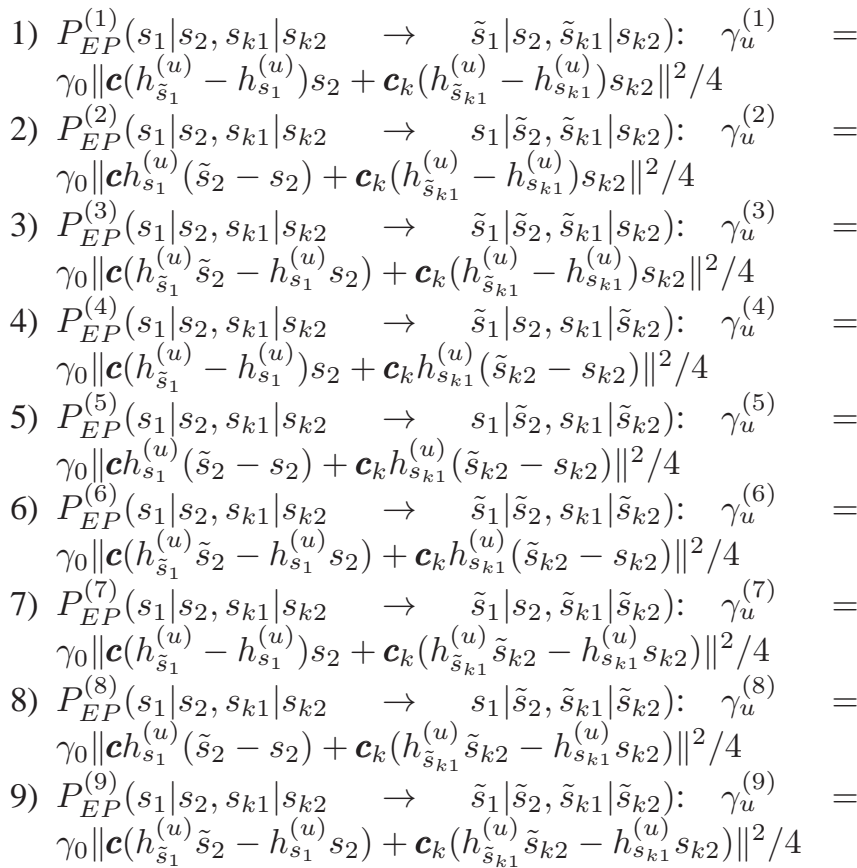

In the above expression, the indices of $m_{i}$ or $\tilde{m}_{i}$ are removed for notational simplicity. The same notational simplifications are used in our forthcoming discourse, whenever there is no confusion. Furthermore, in the case of, such as PEP, which is independent of the specific index, such as a specific TA, there is also no index for $m_{i}$ or $\tilde{m}_{i}$.

As seen in (38) or the above formulas corresponding to the nine cases, the instantaneous SNR $\gamma_{u}^{(i)}$ is a function of the linear combination of 2-to-4 independent Gaussian random variables $h_{\tilde{s}_{1}}^{(u)}, h_{s_{1}}^{(u)}, h_{\tilde{s}_{k 1}}^{(u)}$ and/or $h_{s_{k 1}}^{(u)}$. Hence, for given $\boldsymbol{c}$, $c_{k}, s_{2}, \tilde{s}_{2}, s_{k 2}$ and $\tilde{s}_{k 2}$, the SNR $\gamma_{u}^{(i)}$ obeys the Gamma distribution with the PDF expressed in the form of $[54,56]$

$$
\begin{aligned}
f_{\gamma_{u}^{(i)}}(x)= & \frac{1}{\Gamma\left(\beta_{i}\right)}\left(\frac{\beta_{i}}{\bar{\gamma}_{i}}\right)^{\beta_{i}} x^{\beta_{i}-1} \exp \left(-\frac{\beta_{i} x}{\bar{\gamma}_{i}}\right), \\
& 0 \leq x<\infty
\end{aligned}
$$

where the average $\operatorname{SNR} \bar{\gamma}_{i}$ and the shaping parameter $\beta_{i}$ are determined by the first and second order moments of $\gamma_{u}^{(i)}$, given by

$$
\bar{\gamma}_{i}=E\left[\gamma_{u}^{(i)}\right], \beta_{i}=\frac{\bar{\gamma}_{i}^{2}}{E\left[\left(\gamma_{u}^{(i)}\right)^{2}\right]-\bar{\gamma}_{i}^{2}}
$$

In order to compute $\bar{\gamma}_{i}$ and $\beta_{i}$ with respect to the different cases, we need the second and fourth order moments of complex Gaussian random variables. Let us assume that $X=a+j b$, where both $a$ and $b$ are Gaussian distributed with zero mean and a variance of $\sigma^{2}$. Then, we have $E\left[X^{2}\right]=2 \sigma^{2}$ and $E\left[X^{4}\right]=8 \sigma^{2}$. With these results in mind, we can readily derive $\bar{\gamma}_{i}$ and $\beta_{i}$ for the nine cases, which are expressed respectively as

$$
\begin{aligned}
& \bar{\gamma}_{1}=\gamma_{0}\left(\left|s_{2}\right|^{2}+\left|s_{k 2}\right|^{2}\right) / 2 \\
& \beta_{1}=\frac{\left(\left|s_{2}\right|^{2}+\left|s_{k 2}\right|^{2}\right)^{2}}{\left|s_{2}\right|^{4}+\left|s_{k 2}\right|^{4}+2\left|\rho_{k}\right|^{2}\left|s_{2}\right|^{2}\left|s_{k 2}\right|^{2}} ; \\
& \bar{\gamma}_{2}=\gamma_{0}\left(\left|\tilde{s}_{2}-s_{2}\right|^{2}+2\left|s_{k 2}\right|^{2}\right) / 4 \\
& \beta_{2}=\frac{\left(\left|\tilde{s}_{2}-s_{2}\right|^{2}+2\left|s_{k 2}\right|^{2}\right)^{2}}{\left|\tilde{s}_{2}-s_{2}\right|^{4}+4\left|s_{k 2}\right|^{4}+4\left|\rho_{k}\right|^{2}\left|\tilde{s}_{2}-s_{2}\right|^{2}\left|s_{k 2}\right|^{2}} ; \quad \text { (42a) } \\
& \bar{\gamma}_{3}=\gamma_{0}\left(\left|\tilde{s}_{2}\right|^{2}+\left|s_{2}\right|^{2}+2\left|s_{k 2}\right|^{2}\right) / 4 \\
& \beta_{3}=\frac{\left(\left|\tilde{s}_{2}\right|^{2}+\left|s_{2}\right|^{2}+2\left|s_{k 2}\right|^{2}\right)^{2}}{\left(\left|\tilde{s}_{2}\right|^{2}+s_{2}^{2}\right)^{2}+4\left|s_{k 2}\right|^{4}+4\left|\rho_{k}\right|^{2}\left(\left|\tilde{s}_{2}\right|^{2}+\left|s_{2}\right|^{2}\right)\left|s_{k 2}\right|^{2}}
\end{aligned}
$$

$\bar{\gamma}_{4}=\gamma_{0}\left(2\left|s_{2}\right|^{2}+\left|\tilde{s}_{k 2}-s_{k 2}\right|^{2}\right) / 4$,

$\beta_{4}=\frac{\left(2\left|s_{2}\right|^{2}+\left|\tilde{s}_{k 2}-s_{k 2}\right|^{2}\right)^{2}}{4\left|s_{2}\right|^{4}+\left|\tilde{s}_{k 2}-s_{k 2}\right|^{4}+4\left|\rho_{k}\right|^{2}\left|s_{2}\right|^{2}\left|\tilde{s}_{k 2}-s_{k 2}\right|^{2}}$

$\bar{\gamma}_{5}=\gamma_{0}\left(\left|\tilde{s}_{2}-s_{2}\right|^{2}+\left|\tilde{s}_{k 2}-s_{k 2}\right|^{2}\right) / 4$,

$\beta_{5}=\frac{\left(\left|\tilde{s}_{2}-s_{2}\right|^{2}+\left|\tilde{s}_{k 2}-s_{k 2}\right|^{2}\right)^{2}}{\left|\tilde{s}_{2}-s_{2}\right|^{4}+\left|\tilde{s}_{k 2}-s_{k 2}\right|^{4}+2\left|\rho_{k}\right|^{2}\left|\tilde{s}_{2}-s_{2}\right|^{2}\left|\tilde{s}_{k 2}-s_{k 2}\right|^{2}} ;$

$\bar{\gamma}_{6}=\gamma_{0}\left(\left|\tilde{s}_{2}\right|^{2}+\left|s_{2}\right|^{2}+\left|\tilde{s}_{k 2}-s_{k 2}\right|^{2}\right) / 4$

$\beta_{6}=\frac{\left(\left|\tilde{s}_{2}\right|^{2}+\left|s_{2}\right|^{2}+\left|\tilde{s}_{k 2}-s_{k 2}\right|^{2}\right)^{2}}{\left(\left|\tilde{s}_{2}\right|^{2}+\left|s_{2}\right|^{2}\right)^{2}+\left|\tilde{s}_{k 2}-s_{k 2}\right|^{4}} ;$

$+2\left|\rho_{k}\right|^{2}\left(\left|\tilde{s}_{2}\right|^{2}+\left|s_{2}\right|^{2}\right)\left|\tilde{s}_{k 2}-s_{k 2}\right|^{2}$

$\bar{\gamma}_{7}=\gamma_{0}\left(2\left|s_{2}\right|^{2}+\left|\tilde{s}_{k 2}\right|^{2}+\left|s_{k 2}\right|^{2}\right) / 4$,

$\beta_{7}=\frac{\left(2\left|s_{2}\right|^{2}+\left|\tilde{s}_{k 2}\right|^{2}+\left|s_{k 2}\right|^{2}\right)^{2}}{4\left|s_{2}\right|^{4}+\left(\left|\tilde{s}_{k 2}\right|^{2}+\left|s_{k 2}\right|^{2}\right)^{2}} ;$

$+4\left|\rho_{k}\right|^{2}\left|s_{2}\right|^{2}\left(\left|\tilde{s}_{k 2}\right|^{2}+\left|s_{k 2}\right|^{2}\right)$

$\bar{\gamma}_{8}=\gamma_{0}\left(\left|\tilde{s}_{2}-s_{2}\right|^{2}+\left|\tilde{s}_{k 2}\right|^{2}+\left|s_{k 2}\right|^{2}\right) / 4$,

$\beta_{8}=\frac{\left(\left|\tilde{s}_{2}-s_{2}\right|^{2}+\left|\tilde{s}_{k 2}\right|^{2}+\left|s_{k 2}\right|^{2}\right)^{2}}{\left|\tilde{s}_{2}-s_{2}\right|^{4}+\left(\left|\tilde{s}_{k 2}\right|^{2}+\left|s_{k 2}\right|^{2}\right)^{2}} ;$

$+2\left|\rho_{k}\right|^{2}\left|\tilde{s}_{2}-s_{2}\right|^{2}\left(\left|\tilde{s}_{k 2}\right|^{2}+\left|s_{k 2}\right|^{2}\right)$

$\bar{\gamma}_{9}=\gamma_{0}\left(\left|\tilde{s}_{2}\right|^{2}+\left|s_{2}\right|^{2}+\left|\tilde{s}_{k 2}\right|^{2}+\left|s_{k 2}\right|^{2}\right) / 4$

$$
\begin{aligned}
\beta_{9}= & \frac{\left(\left|\tilde{s}_{2}\right|^{2}+\left|s_{2}\right|^{2}+\left|\tilde{s}_{k 2}\right|^{2}+\left|s_{k 2}\right|^{2}\right)^{2}}{\left(\left|\tilde{s}_{2}\right|^{2}+\left|s_{2}\right|^{2}\right)^{2}+\left(\left|\tilde{s}_{k 2}\right|^{2}+\left|s_{k 2}\right|^{2}\right)^{2}} \\
& +2\left|\rho_{k}\right|^{2}\left(\left|\tilde{s}_{2}\right|^{2}+\left|s_{2}\right|^{2}\right)\left(\left|\tilde{s}_{k 2}\right|^{2}+\left|s_{k 2}\right|^{2}\right)
\end{aligned}
$$

In the above equations, $\rho_{k}=\boldsymbol{c}^{H} \boldsymbol{c}_{k}$ is the cross-correlation between $\boldsymbol{c}$ and $\boldsymbol{c}_{k}$. From $\bar{\gamma}_{i}$ and $\beta_{i}$ shown in (42a) - (42i), it is not hard for us to infer the following observations:

- In the formulas (42a) - (42i), when we set $\left|\tilde{s}_{k 2}\right|^{2}=0$ and $\left|s_{k 2}\right|^{2}=0$, we always obtain $\beta_{i}=1$ for $i=1, \ldots, 9$. Correspondingly, the nine cases are reduced to the three cases, which we considered earlier in Section IV-A for the single-user scenario or that all the $(K-1)$ interfering users are correctly detected.

- $\bar{\gamma}_{i}$ is always increased due to the error event of a second user, meaning that having two users simultaneously in error is rare compared to having a single user in error in a SM-SCDMA system.

- As $0 \leq\left|\rho_{k}\right|<1$, we can find $1<\beta_{i} \leq 2$ for all the nine cases. Since a higher $\beta_{i}$ value results in a lower error probability, as seen in $[54,56]$ and below, again, this explains that the probability of two users being simultaneously in error is lower than that of only one user 
being in error.

- When the erroneous user $k$ does not share any chips with the reference user, we then have $\rho_{k}=0$. Consequently, the value of $\beta_{i}$ becomes higher than that of the case, where the erroneous user $k$ and the reference user share some chips for transmission. Therefore, an interfering user not sharing chips with the reference user imposes a lower impact on the error probability of the reference user than an interfering user sharing some chips with the reference user.

Having obtained $\bar{\gamma}_{i}$ and $\beta_{i}$ for the different cases, the corresponding PEPs can be obtained via averaging (37) using (40), yielding $[54,56]$

$$
\begin{aligned}
P_{E P}^{(i)}\left(x, x_{k} \rightarrow \tilde{x}, \tilde{x}_{k}\right)= & \frac{1}{\pi} \int_{0}^{\pi / 2}\left(\frac{\beta_{i} \sin ^{2} \theta}{\beta_{i} \sin ^{2} \theta+\bar{\gamma}_{i}}\right)^{\beta_{i} U} d \theta \\
& i=1, \ldots, 9
\end{aligned}
$$

which, according to $[54,56-58]$, can be represented in a closed-form as

$$
\begin{aligned}
& P_{E P}^{(i)}\left(x, x_{k} \rightarrow \tilde{x}, \tilde{x}_{k}\right) \\
& =\frac{\Gamma\left(\beta_{i} U+1 / 2\right)}{2 \sqrt{\pi} \Gamma\left(\beta_{i} U+1\right)} \sqrt{\frac{\bar{\gamma}_{i}}{\beta_{i}+\bar{\gamma}_{i}}}\left(\frac{\beta_{i}}{\beta_{i}+\bar{\gamma}_{i}}\right){ }^{\beta_{i} U} \\
& \quad \times{ }_{2} F_{1}\left(1, \beta_{i} U+\frac{1}{2} ; \beta_{i} U+1 ; \frac{\beta_{i}}{\beta_{i}+\bar{\gamma}_{i}}\right), \\
& \quad i=1, \ldots, 9
\end{aligned}
$$

where ${ }_{2} F_{1}(a, b ; c ; z)$ is the hypergeometric function defined as [59] ${ }_{2} F_{1}(a, b ; c ; z)=\sum_{k=0}^{\infty} \frac{(a)_{k}(b)_{k} z^{k}}{(c)_{k} k !}$ and $(a)_{k}=a(a+$ 1) $\cdots(a+k-1),(a)_{0}=1$.

Below we provide the expressions for computing the approximate error probabilities in some special cases of practical interest.

First, for the ABER of the SM-SCDMA systems supporting $K$ users (one reference user plus $(K-1)$ interfering users), we assume that there is at most one interfering user in error. This assumption is reasonable due to the fact that when the SNR is sufficiently high, the probability of having two or more users simultaneously detected in error in addition to the reference user is negligible. For example, let us assume that the error rate of a user is about $10^{-3}$, which is the error rate of interest in practice. Then, when an optimum detector is employed, the average number of users erroneously detected during a channel use is about $K \times 10^{-3}$, provided that $K$ is comparable to the total number of chips used by the SM-SCDMA system. However, we should note that when there are two or more users simultaneously detected in error, the erroneous events of these users generate some correlation, as implied by (42a) - (42i). Nevertheless, this correlation should not significantly affect the average number of erroneous users, because the probability of having two or more users simultaneously in error is insignificant, in comparison to that of having only one user in error. Based on the above assumption and (35), the ABER of the SM-SCDMA systems can be expressed as

$$
\begin{aligned}
\bar{P}_{b M} \approx & \left(1-\bar{P}_{s S}\right)^{K-1} \bar{P}_{b S}+\left[1-\left(1-\bar{P}_{s S}\right)^{K-1}\right] \\
& \times \frac{1}{K-1} \sum_{k=1}^{K-1} \bar{P}_{b M}(k)
\end{aligned}
$$

where $\bar{P}_{b M}(k)$ is the ABER of the reference user when the $k$ th interfering user is in error, which is given by (39). When taking into account our nine cases, we have

$$
\begin{aligned}
& \bar{P}_{b M}(k) \approx \frac{M_{1}^{2}\left(M_{1}-1\right) b_{1}}{2 M_{2} b} \sum_{m_{2}=0}^{M_{2}-1} \sum_{m_{k 2}=0}^{M_{2}-1} \\
& \times P_{E P}\left(s_{1}\left|s_{2}^{\left(m_{2}\right)}, s_{k 1}\right| s_{k 2}^{\left(m_{k 2}\right)} \rightarrow \tilde{s}_{1}\left|s_{2}^{\left(m_{2}\right)}, \tilde{s}_{k 1}\right| s_{k 2}^{\left(m_{k 2}\right)}\right) \\
& +\frac{M_{1}\left(M_{1}-1\right)}{M_{2} b} \sum_{m_{2}=0}^{M_{2}-1} \sum_{\tilde{m}_{2} \neq m_{2}}^{M_{2}-1} \sum_{m_{k 2}=0}^{M_{2}-1} D\left(\boldsymbol{b}_{2}^{\left(m_{2}\right)}, \tilde{\boldsymbol{b}}_{2}^{\left(\tilde{m}_{2}\right)}\right) \\
& \times P_{E P}\left(s_{1}\left|s_{2}^{\left(m_{2}\right)}, s_{k 1}\right| s_{k 2}^{\left(m_{k 2}\right)} \rightarrow s_{1}\left|\tilde{s}_{2}^{\left(\tilde{m}_{2}\right)}, \tilde{s}_{k 1}\right| s_{k 2}^{\left(m_{k 2}\right)}\right) \\
& +\frac{M_{1}\left(M_{1}-1\right)}{M_{2} b} \sum_{m_{2}=0}^{M_{2}-1} \sum_{\tilde{m}_{2} \neq m_{2}}^{M_{2}-1} \sum_{m_{k 2}=0}^{M_{2}-1}\left[\frac{b_{1} M_{1}}{2}+\left(M_{1}-1\right)\right. \\
& \left.\times D\left(\boldsymbol{b}_{2}^{\left(m_{2}\right)}, \tilde{\boldsymbol{b}}_{2}^{\left(\tilde{m}_{2}\right)}\right)\right] \\
& \times P_{E P}\left(s_{1}\left|s_{2}^{\left(m_{2}\right)}, s_{k 1}\right| s_{k 2}^{\left(m_{k 2}\right)} \rightarrow \tilde{s}_{1}\left|\tilde{s}_{2}^{\left(\tilde{m}_{2}\right)}, \tilde{s}_{k 1}\right| s_{k 2}^{\left(m_{k 2}\right)}\right) \\
& +\frac{M_{1}^{2} b_{1}}{2 M_{2} b} \sum_{m_{2}=0}^{M_{2}-1} \sum_{m_{k 2}=0}^{M_{2}-1} \sum_{\tilde{m}_{k 2} \neq m_{k 2}}^{M_{2}-1} P_{E P}\left(s_{1}\left|s_{2}^{\left(m_{2}\right)}, s_{k 1}\right| s_{k 2}^{\left(m_{k 2}\right)}\right. \\
& \left.\rightarrow \tilde{s}_{1}\left|s_{2}^{\left(m_{2}\right)}, s_{k 1}\right| \tilde{s}_{k 2}^{\left(\tilde{m}_{k 2}\right)}\right) \\
& +\frac{M_{1}}{M_{2} b} \sum_{m_{2}=0}^{M_{2}-1} \sum_{\tilde{m}_{2}=\neq m_{2}}^{M_{2}-1} \sum_{m_{k 2}=0}^{M_{2}-1} \sum_{\tilde{m}_{k 2} \neq m_{k 2}}^{M_{2}-1} D\left(\boldsymbol{b}_{2}^{\left(m_{2}\right)}, \tilde{\boldsymbol{b}}_{2}^{\left(\tilde{m}_{2}\right)}\right) \\
& \times P_{E P}\left(s_{1}\left|s_{2}^{\left(m_{2}\right)}, s_{k 1}\right| s_{k 2}^{\left(m_{k 2}\right)} \rightarrow s_{1}\left|\tilde{s}_{2}^{\left(\tilde{m}_{2}\right)}, s_{k 1}\right| \tilde{s}_{k 2}^{\left(\tilde{m}_{k 2}\right)}\right) \\
& +\frac{M_{1}}{M_{2} b} \sum_{m_{2}=0}^{M_{2}-1} \sum_{\tilde{m}_{2} \neq m_{2}}^{M_{2}-1} \sum_{m_{k 2}=0}^{M_{2}-1} \sum_{\tilde{m}_{k 2} \neq m_{k 2}}^{M_{2}-1}\left[\frac{b_{1} M_{1}}{2}+\left(M_{1}-1\right)\right. \\
& \left.\times D\left(\boldsymbol{b}_{2}^{\left(m_{2}\right)}, \tilde{\boldsymbol{b}}_{2}^{\left(\tilde{m}_{2}\right)}\right)\right] \\
& \times P_{E P}\left(s_{1}\left|s_{2}^{\left(m_{2}\right)}, s_{k 1}\right| s_{k 2}^{\left(m_{k 2}\right)} \rightarrow \tilde{s}_{1}\left|\tilde{s}_{2}^{\left(\tilde{m}_{2}\right)}, s_{k 1}\right| \tilde{s}_{k 2}^{\left(\tilde{m}_{k 2}\right)}\right) \\
& +\frac{M_{1}^{2}\left(M_{1}-1\right) b_{1}}{2 M_{2} b} \sum_{m_{2}=0}^{M_{2}-1} \sum_{m_{k 2}=0}^{M_{2}-1} \sum_{\tilde{m}_{k 2} \neq m_{k 2}}^{M_{2}-1} P_{E P}\left(s_{1} \mid s_{2}^{\left(m_{2}\right)},\right. \\
& \left.\times s_{k 1}\left|s_{k 2}^{\left(m_{k 2}\right)} \rightarrow \tilde{s}_{1}\right| s_{2}^{\left(m_{2}\right)}, \tilde{s}_{k 1} \mid \tilde{s}_{k 2}^{\left(\tilde{m}_{k 2}\right)}\right) \\
& +\frac{M_{1}\left(M_{1}-1\right)}{M_{2} b} \sum_{m_{2}=0}^{M_{2}-1} \sum_{\tilde{m}_{2} \neq m_{2}}^{M_{2}-1} \sum_{m_{k 2}=0}^{M_{2}-1} \sum_{\tilde{m}_{k 2} \neq m_{k 2}}^{M_{2}-1} D\left(\boldsymbol{b}_{2}^{\left(m_{2}\right)}, \tilde{\boldsymbol{b}}_{2}^{\left(\tilde{m}_{2}\right)}\right) \\
& \times P_{E P}\left(s_{1}\left|s_{2}^{\left(m_{2}\right)}, s_{k 1}\right| s_{k 2}^{\left(m_{k 2}\right)} \rightarrow s_{1}\left|\tilde{s}_{2}^{\left(\tilde{m}_{2}\right)}, \tilde{s}_{k 1}\right| \tilde{s}_{k 2}^{\left(\tilde{m}_{k 2}\right)}\right) \\
& +\frac{M_{1}\left(M_{1}-1\right)}{M_{2} b} \sum_{m_{2}=0}^{M_{2}-1} \sum_{\tilde{m}_{2} \neq m_{2}}^{M_{2}-1} \sum_{m_{k 2}=0}^{M_{2}-1} \sum_{\tilde{m}_{k 2} \neq m_{k 2}}^{M_{2}-1}\left[\frac{b_{1} M_{1}}{2}\right. \\
& \left.+\left(M_{1}-1\right) D\left(\boldsymbol{b}_{2}^{\left(m_{2}\right)}, \tilde{\boldsymbol{b}}_{2}^{\left(\tilde{m}_{2}\right)}\right)\right] \\
& \times P_{E P}\left(s_{1}\left|s_{2}^{\left(m_{2}\right)}, s_{k 1}\right| s_{k 2}^{\left(m_{k 2}\right)} \rightarrow \tilde{s}_{1}\left|\tilde{s}_{2}^{\left(\tilde{m}_{2}\right)}, \tilde{s}_{k 1}\right| \tilde{s}_{k 2}^{\left(\tilde{m}_{k 2}\right)}\right)
\end{aligned}
$$


Note that, in (46), all the PEPs are in the form of (43) or (44), with the corresponding $\bar{\gamma}_{i}$ and $\beta_{i}$ are respectively given in (42a) - (42i), which are determined by the specific symbols of $s_{2}^{\left(m_{2}\right)}, s_{k 2}^{\left(m_{k 2}\right)}, \tilde{s}_{2}^{\left(\tilde{m}_{2}\right)}, \tilde{s}_{k 2}^{\left(\tilde{m}_{k 2}\right)}$ as well as $\rho_{k}$ of the crosscorrelation between the sparse codes of the reference user and of the $k$ th interfering user.

Secondly, in order to reduce the computations invoked, another ABER expression can be obtained by considering only the specific interfering users sharing at least one chip with the reference user. In this case, the ABER can be expressed as

$$
\begin{aligned}
\bar{P}_{b M}^{\prime} \approx & \left(1-\bar{P}_{s S}\right)^{K-1} \bar{P}_{b S}+\left[1-\left(1-\bar{P}_{s S}\right)^{K-1}\right] \\
& \times \frac{1}{K-1} \sum_{k \in \mathcal{K}} \bar{P}_{b M}(k)
\end{aligned}
$$

where $\mathcal{K}=\left\{k \mid \mathcal{C}_{k} \cap \mathcal{C} \neq \varnothing \forall k=1,2, \ldots, K-1\right\}$, and $\varnothing$ is an empty set.

Thirdly, we are interested in the ABER of the reference user, when there is a single erroneous interfering user and this user does not share any chips with the reference user. This ABER can be formulated as

$$
\bar{P}_{I M} \approx \bar{P}_{b M}(k)
$$

where $k \notin \mathcal{K}$ can be any arbitrary user not in $\mathcal{K}$. Note that any $k \notin \mathcal{K}$ has the same impact on the reference user.

Finally, the ABER of the reference user on condition that there is only a single erroneous interfering user and this user shares some chips with the reference user can be expressed as

$$
\bar{P}_{I M}^{\prime} \approx \frac{1}{\sum 1_{k \in \mathcal{K}}} \sum_{k \in \mathcal{K}} \bar{P}_{b M}(k)
$$

where $\sum 1_{k \in \mathcal{K}}$ denotes the total number of the interfering users sharing some chips with the reference user. Note that when regular sparse codes are employed, implying that the number of chips shared between any interfering user in $\mathcal{K}$ with the reference user is the same, then the averaging operation in (49) is not required. Instead, we can compute $\bar{P}_{I M}^{\prime}$ by considering a single interfering user randomly chosen from $\mathcal{K}$.

\section{Performance Results}

In this section, both the theoretical error probability bounds and the simulation results of SM-SCDMA systems are presented. Firstly, we propose a new 8QAM constellation, which is then considered in some of the other figures. Then, the performance of SM-SCDMA systems associated with various parameters are characterized, based on which we also find the valid range of our formulas derived. Finally, we study the performance of relatively large-scale SM-SCDMA systems, which are impervious to numerical simulations. Note that the parameters used for generating the results of a specific figure are directly detailed in conjunction with the figure.

Again, let us propose a new 8QAM constellation for our forthcoming investigation. In the literature, typically the three 8QAM constellations of Fig. 3(b)-(d) are considered, all of which facilitate Gray coding [60]. Specifically, the constellation of Fig. 3(b) is designed based on the classic square-16QAM. It has a peak-to-average ratio (PAR) of 1.8, and the minimum distance of $0.63 \sqrt{E_{0}}$, where $E_{0}$ is the average phasor energy [60]. Here, we propose a new 8QAM constellation also designed based on square-16QAM, as shown in Fig. 3(a). It can be shown that this new constellation has the same PAR as that shown in Fig. 3(b), but a minimum distance of $0.88 \sqrt{E_{0}}$. However, the Gray coding cannot be fully applied, as each 8QAM symbol has only three bits, but there are two points, namely, '000' and '010', each having four neighbors.
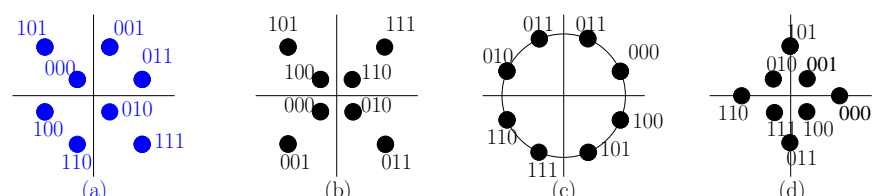

Fig. 3. Different constellations for 8QAM.

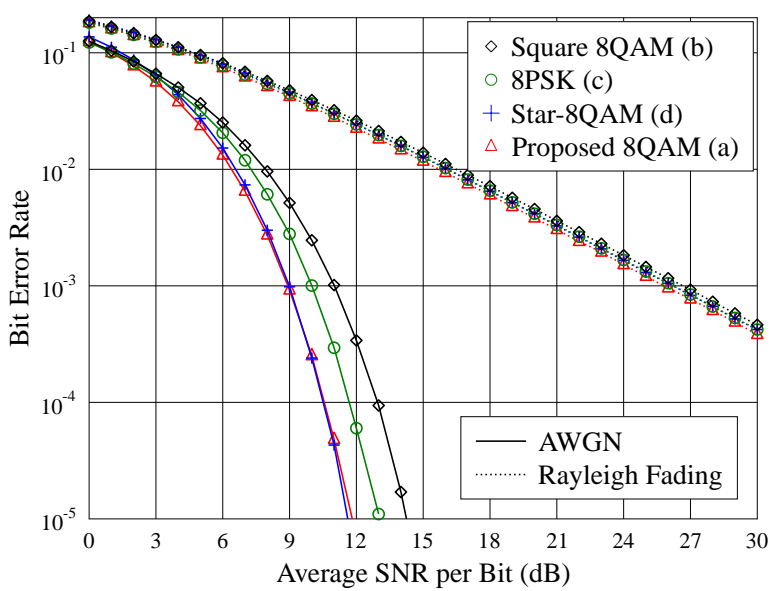

Fig. 4. BER performance of different 8QAM constellations over Gaussian channels.

The BER performance of the above four 8QAM constellations when communicating over Gaussian or Rayleigh fading channels is depicted in Fig. 4. Explicitly, in Gaussian channels, the proposed 8QAM scheme marginally outperforms all the others 8QAM schemes, when the SNR is lower than about $10 \mathrm{~dB}$, i.e. in the SNR range of interest. By contrast, when considering Rayleigh fading channels, the proposed 8QAM scheme slightly outperforms the others 8QAM schemes across the whole SNR region considered. Note that since the proposed 8QAM constellation is embedded in the 16QAM constellation, it is beneficial for the implementation of adaptive modulation [61] in practice. Therefore, in our following results, we assume the constellation of Fig. 3(a), when 8QAM is employed.

Fig. 5 shows the BER performance of SM-SCDMA systems, when different number of modulation levels are assumed for the $M_{1}$ SSK and $M_{2} \mathrm{QAM}$. In this figure, we also compare the results obtained by simulations and those evaluated from the ABER bounds of (45) and (47). Furthermore, the singleuser ABER bound of (27) is provided. Based on the results, we have the following observations. Firstly, when the throughput 


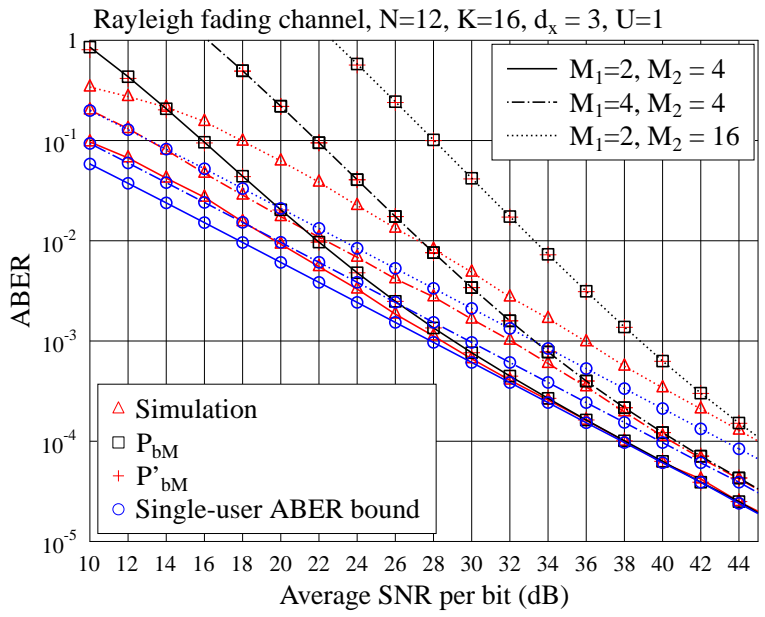

Fig. 5. ABER performance of SM-SCDMA systems for different number of modulation levels over Rayleigh fading channels.

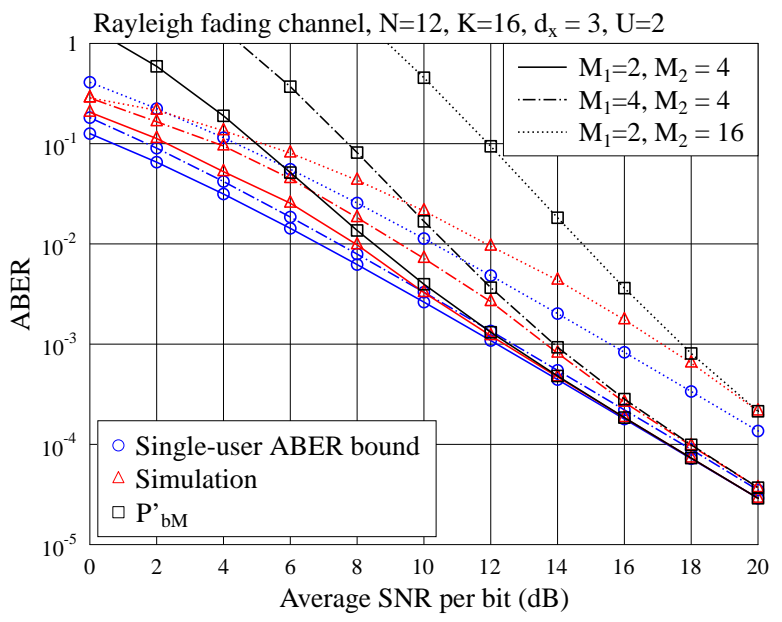

Fig. 6. ABER performance of SM-SCDMA systems with different number of modulation levels over Rayleigh fading channels.

is increased either by increasing $M_{1}$ or $M_{2}$, the BER performance degrades. Secondly, when the SNR increases, the BER performance of SM-SCDMA system using $N=12$ chips to support $K=16$ users, which corresponds to a normalized user-load factor of $4 / 3$, converges to the singleuser BER. Thirdly, the ABER bound evaluated by (45) and that evaluated by (47) are indistinguishable, which requires much less computation than (45). Furthermore, as expected, at the BER of about $10^{-3}$, the ABER bounds evaluated from (45) or (47) are close to the corresponding results obtained via simulations, and they become closer, as the SNR increases. Therefore, when the SNR is sufficiently high, resulting in a BER of about $10^{-3}$ or lower, the single user ABER bound of (27) and the ABER bound of (45) or (47) can be used to predict the achievable performance of SM-SCDMA systems.

In Fig. 5, we assumed $U=1$. By contrast, we assume $U=$ 2 in Fig. 6. The other parameters are the same for both figures. By comparing the results of these two figures, we can see that a significant performance improvement is available, when the number of receive antennas is increased from $U=1$ to $U=2$, which is an explicit benefit of the diversity gain and the power gain. Furthermore, similar to Fig. 5, the BER obtained via simulations lies between the single-user ABER bound of (27) and the ABER bound of (47) (or (45)). Therefore, we may use (27) and (47) to predict the BER performance of SM-SCDMA systems, or simply use (47), when the SNR is sufficiently high, resulting in a BER of $10^{-3}$ or lower.

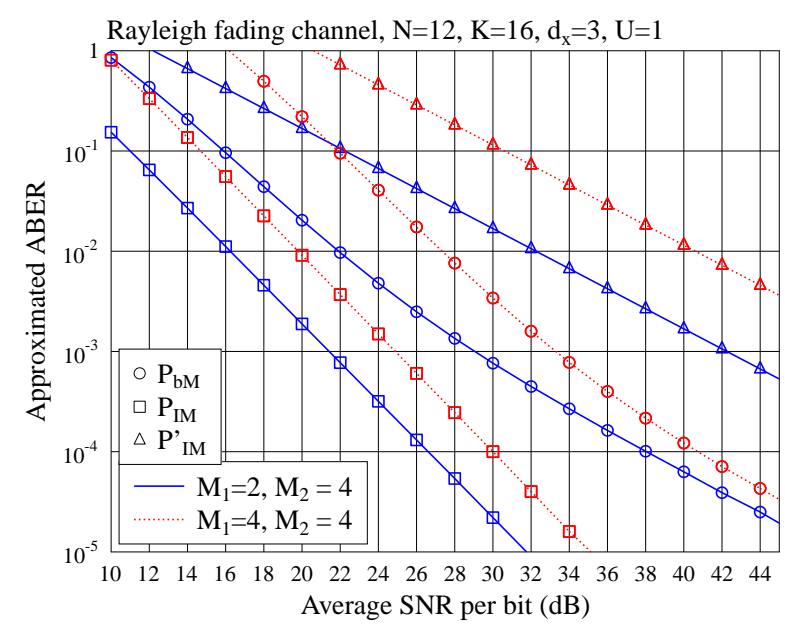

Fig. 7. Comparison of the conditional ABER of $\bar{P}_{I M}$ and $\bar{P}_{I M}^{\prime}$, and the ABER of $\bar{P}_{b M}$ of SM-SCDMA systems.

In Section IV, we have considered the ABER $\bar{P}_{I M}$ of a reference user, when there is a single erroneous interfering user without overlapping with the reference user, which is expressed in (48). We have also considered the ABER $\bar{P}_{I M}^{\prime}$ of a reference user, when there is a single erroneous interfering user sharing some chips with the reference user, which is expressed in (49). In Fig. 7, we compare these two conditional ABERs as well as $P_{b M}$ of (45). The results show that for a given SNR, $\bar{P}_{I M}^{\prime}$ is much higher than $\bar{P}_{I M}$, implying that the ABER is dominated by those specific erroneous interfering users, which share some chips with the reference user. This also explain that in Figs. 5, $P_{b M}$ and $P_{b M}^{\prime}$ are indistinguishable.

Fig. 8 depicts the performance of SM-SCDMA systems for the user-load factors of $K=16,20$ and 24, giving the normalized user-load factors of $4 / 3,5 / 3$ and 2 . In addition to the observations from the previous figures, Fig. 8 shows that for the other parameters considered, the performance of the SCDMA systems only slightly degrades, as the number of users increases. This is the case, in particular when a SMSCDMA system employs $U=2$ receive antennas. As shown in Fig. 8, a relatively substantial performance drop is observed, when the normalized user-load factor is changed from $4 / 3$ to $5 / 3$. By contrast, there is only a marginal performance loss, when the normalized user-load factor is increased from $5 / 3$ to 2 .

Again, SM-SCDMA systems rely on two types of modulations schemes, i.e. the SSK and QAM. While SSK belongs to the family of energy-efficient modulation schemes, QAM is a bandwidth-efficient modulation scheme [55]. Hence, when fixing the bandwidth, there should be a trade-off between the 


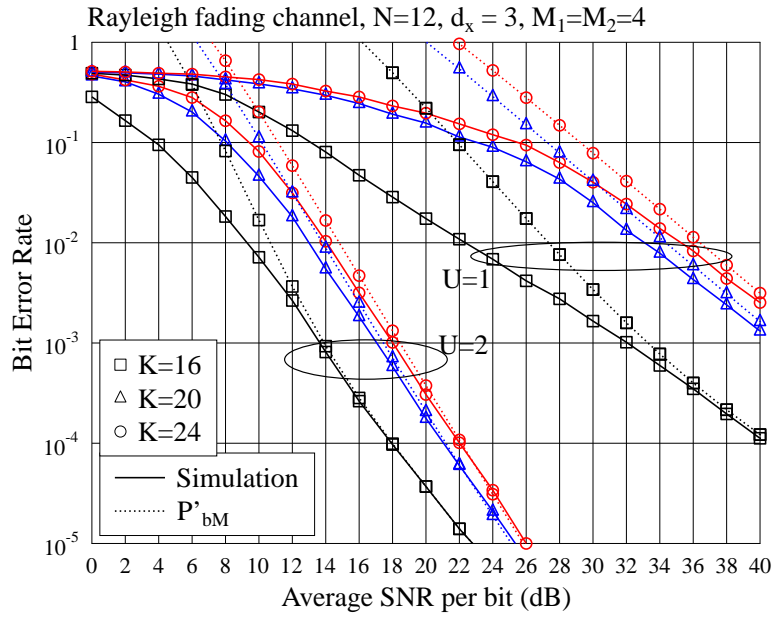

Fig. 8. ABER performance of SM-SCDMA systems for different user-loads.

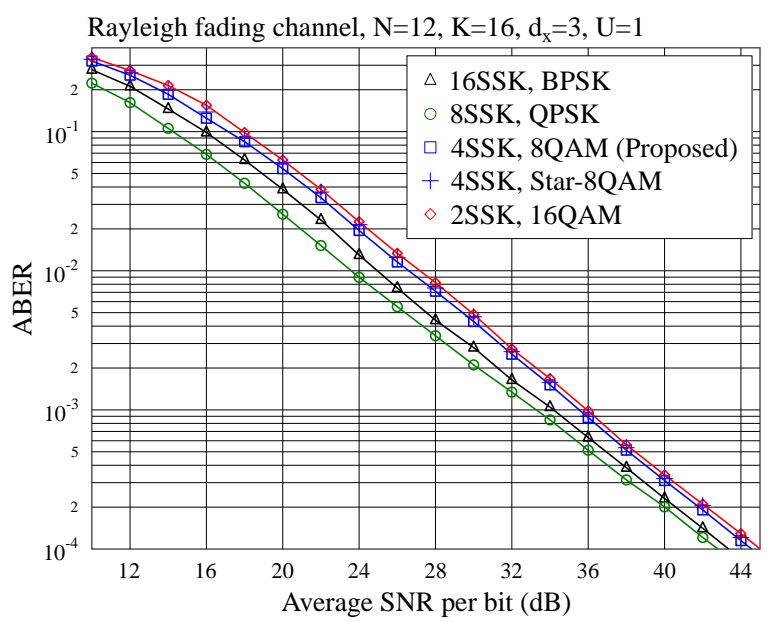

Fig. 9. ABER performance of the SM-SCDMA system for different modulation schemes transmitting $b=5$ bits per symbol.

numbers of bits allocated to these two modulation schemes. Therefore, in Fig. 9, we demonstrate this trade-off, when assuming that the total number of bits per symbol is fixed to 5 . Explicitly, the combination of 8SSK and QPSK achieves the best error performance among the four possible combinations over the whole SNR region considered. Additionally, in Fig. 9 the proposed 8QAM shows slightly better ABER performance than the star 8QAM, when communicating over Rayleigh fading channels. This is also reflected in Fig. 9, where the proposed 8QAM scheme slightly outperforms the star 8QAM operating in Rayleigh fading channels over the whole SNR range considered.

So far, we have considered some relatively small SMSCDMA systems associated with $N=12$, so that we can use simulation results to validate our mathematical analysis and gain insights into the characteristics of the various expressions derived. Below we consider some relatively large-scale SMSCDMA systems, whose performance is infeasible to study by Monte-Carlo simulations.

In Figs. 10 and 11, we plot the approximated ABER evalu-

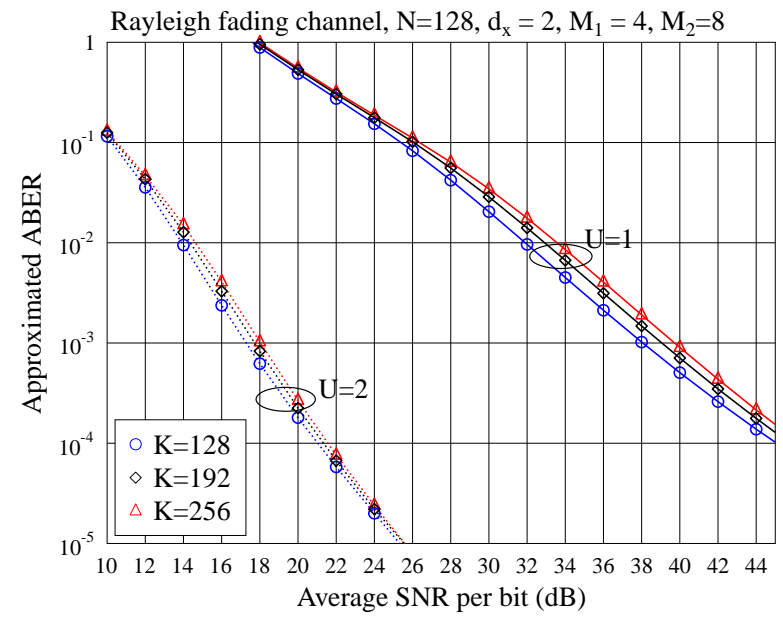

Fig. 10. ABER performance of SM-SCDMA systems for different user-loads.

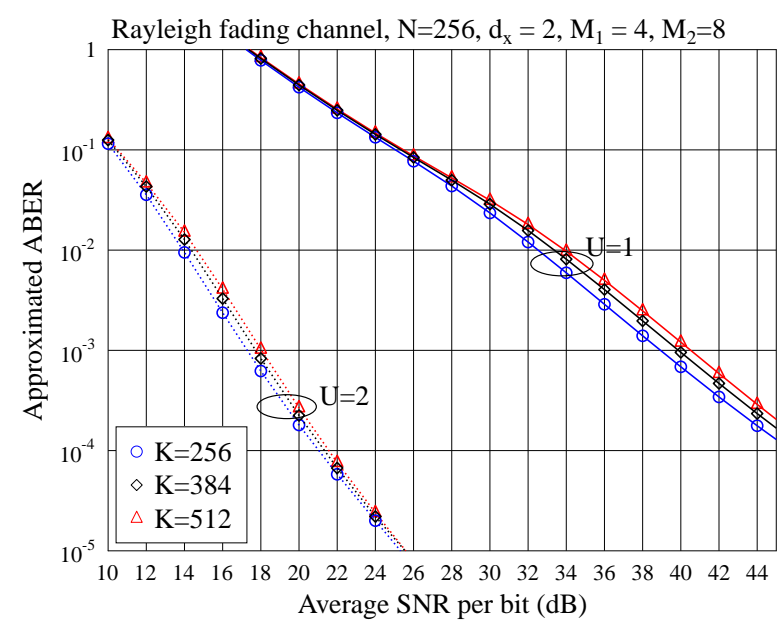

Fig. 11. ABER performance of SM-SCDMA systems for different user-loads

ated from $\bar{P}_{b M}^{\prime}$ of (47) for $N=128$ and 256, respectively. For both cases, we examine the performance, when the normalized user-load factors are respectively $1,1.5$ and 2 . We can see from the results that the error performance curves of all the three loading factors are close to each other. From the results of Figs.8, 10 and 11 we infer that when a SM-SCDMA system becomes larger in terms of $N$, it is capable of supporting a heaver user-load at a given error rate.

\section{Vi. Conclusions}

We have proposed a SM-SCDMA system for supporting high-user-load MA transmission. In order to show the potential of SM-SCDMA and to allow it to achieve low-complexity near-optimum detection, we have considered both the MLD and the MPAD developed based on the MAPD. We have added new ingredients to the error performance analysis of SM systems and analyzed the ABER of SM-SCDMA systems employing MLD. A range of expressions have been obtained for estimating the ABER of SM-SCDMA systems operating in different situations. Finally, the performance of SM-SCDMA 
systems has been investigated based on both simulations and numerical evaluation of the expressions derived. Our studies and performance results demonstrate that SM-SCDMA is capable of supporting large-scale MA. It is also capable of exploiting the space-time domain resources for improving energy efficiency. With the aid of the MAPD, it can support even a normalized user-load factor of 2 without much performance degradation. Furthermore, when a SM-SCDMA system becomes larger in terms of the spreading factor obtained by utilizing more bandwidth, it becomes more efficient, and the performance only slightly degrades, when the normalized userload factor is increased from one to two. Additionally, we note that the error performance achieved by the MAPD is near optimum, which is close to that attained by the MLD.

Our future research will investigate SM-SCDMA on the basis of multicarrier transmission over frequency-selective fading channels.

\section{REFERENCES}

[1] P. Wang, J. Xiao, and L. Ping, "Comparison of orthogonal and nonorthogonal approaches to future wireless wireless cellular systems," IEEE Vehicular Technology Magazine, vol. 1, no. 3, pp. 4 - 11, September 2006

2] R. Hoshyar, F. P. Wathan, and R. Tafazolli, "Novel low-density signature for synchronous CDMA systems over AWGN channel," IEEE Transactions on Signal Processing, vol. 56, no. 4, pp. 1616-1626, 2008.

[3] J. Schaepperle and A. Ruegg, "Enhancement of throughput and fairness in $4 \mathrm{G}$ wireless access systems by non-orthogonal signaling," Bell Labs Technical Journal, vol. 13, no. 4, pp. 59 - 77, 2009.

[4] J. Harshan and B. Rajan, "On two-user Gaussian multiple access channels with finite input constellations," IEEE Transactions on Information Theory, vol. 57, no. 3, pp. 1299-1327, March 2011.

[5] Z. Ding, Z. Yang, P. Fan, and H. V. Poor, "On the performance of non-orthogonal multiple access in $5 \mathrm{G}$ systems with randomly deployed users," IEEE Signal Processing Letters, vol. 21, no. 12, pp. 1501-1505, Dec 2014

[6] L. Dai, B. Wang, Y. Yuan, S. Han, C. 1. I, and Z. Wang, "Non-orthogonal multiple access for 5G: Solutions, challenges, opportunities, and future research trends," IEEE Communications Magazine, vol. 53, no. 9, pp. 74-81, September 2015.

[7] L. Lu, Y. Chen, W. Guo, H. Yang, Y. Wu, and S. Xing, "Prototype for 5G new air interface technology SCMA and performance evaluation,' China Communications, vol. 12, no. Supplement, pp. 38-48, December 2015.

[8] J. B. Kim and I. H. Lee, "Capacity analysis of cooperative relaying systems using non-orthogonal multiple access," IEEE Communications Letters, vol. 19, no. 11, pp. 1949-1952, Nov 2015.

[9] M. Al-Imari, P. Xiao, and M. A. Imran, "Receiver and resource allocation optimization for uplink NOMA in 5G wireless networks," in 2015 International Symposium on Wireless Communication Systems (ISWCS), Aug 2015, pp. 151-155.

[10] F.-L. Luo and C. Zhang, Non-orthogonal Multi-User Superposition and Shared Access. Wiley-IEEE Press, 2016, pp. 616-. [Online]. Available: http://ieeexplore.ieee.org/xpl/articleDetails.jsp?arnumber $=7572709$

[11] J. Men and J. Ge, "Performance analysis of non-orthogonal multiple access in downlink cooperative network," IET Communications, vol. 9 , no. 18 , pp. 2267-2273, 2015.

[12] Z. Ding, R. Schober, and H. V. Poor, "A general MIMO framework for NOMA downlink and uplink transmission based on signal alignment,' IEEE Transactions on Wireless Communications, vol. 15, no. 6, pp. 4438-4454, June 2016.

[13] J. Men, J. Ge, and C. Zhang, "Performance analysis of non-orthogonal multiple access for relaying networks over Nakagami-m fading channels," IEEE Transactions on Vehicular Technology, vol. PP, no. 99, pp. $1-1,2016$.

[14] Y. Zhang, H. M. Wang, T. X. Zheng, and Q. Yang, "Energy-efficient transmission design in non-orthogonal multiple access," IEEE Transactions on Vehicular Technology, vol. PP, no. 99, pp. 1-1, 2016.

[15] X. zhu, Z. Wang and J. Cao, "NOMA-Based Spatial Modulation," IEEE Access, vol. 5, pp. 3790-3800, Mar. 2017.
[16] M. Yoshida and T. Tanaka, "Analysis of sparsely-spread CDMA via statistical mechanics," in 2006 IEEE International Symposium on Information Theory. IEEE, 2006, pp. 2378-2382.

[17] T. Tanaka, "A statistical-mechanics approach to large-system analysis of CDMA multiuser detectors," IEEE Transactions on Information theory, vol. 48, no. 11, pp. 2888-2910, 2002.

[18] D. Guo and C.-C. Wang, "Multiuser detection of sparsely spread CDMA," IEEE Journal on Selected Areas in Communications, vol. 26 , no. 3, pp. 421-431, 2008

[19] H. Nikopour and H. Baligh, "Sparse code multiple access," in 2013 IEEE 24th Annual International Symposium on Personal, Indoor, and Mobile Radio Communications (PIMRC). IEEE, 2013, pp. 332-336.

[20] M. Taherzadeh, H. Nikopour, A. Bayesteh, and H. Baligh, "SCMA codebook design," in Vehicular Technology Conference (VTC Fall), 2014 IEEE 80th. IEEE, 2014, pp. 1-5.

[21] D. Cai, P. Fan, X. Lei, Y. Liu, and D. Chen, "Multi-dimensional SCMA codebook design based on constellation rotation and interleaving," in Vehicular Technology Conference (VTC Spring), 2016 IEEE 83rd. IEEE, 2016, pp. 1-5.

[22] S. Verdu, Multiuser Detection. Cambridge University Press, 1998.

[23] T. J. Richardson and R. L. Urbanke, "The capacity of low-density paritycheck codes under message-passing decoding," IEEE transactions on information theory, vol. 47, no. 2, pp. 599-618, 2001.

[24] F. R. Kschischang, B. J. Frey, and H.-A. Loeliger, "Factor graphs and the sum-product algorithm," IEEE Transactions on information theory, vol. 47, no. 2, pp. 498-519, 2001

[25] J. Van De Beek and B. M. Popovic, "Multiple access with lowdensity signatures," in Global Telecommunications Conference, 2009. GLOBECOM 2009. IEEE. IEEE, 2009, pp. 1-6.

[26] H. Mu, Z. Ma, M. Alhaji, P. Fan, and D. Chen, "A fixed low complexity message pass algorithm detector for up-link SCMA system," IEEE Wireless Communications Letters, vol. 4, no. 6, pp. 585-588, 2015.

[27] R. Y. Mesleh, H. Haas, S. Sinanovic, C. W. Ahn, and S. Yun, "Spatial modulation," IEEE Transactions on Vehicular Technology, vol. 57, no. 4 pp. 2228-2241, 2008.

[28] M. Di Renzo, H. Haas, A. Ghrayeb, S. Sugiura, and L. Hanzo, "Spatial modulation for generalized MIMO: Challenges, opportunities, and implementation," Proceedings of the IEEE, vol. 102, no. 1, pp. 56-103, 2014.

[29] P. Yang, M. D. Renzo, and et.al, "Design guidelines for spatial modulation," IEEE Commun. Sur. \& Tutor, vol. 17, no. 1, pp. 6-26, First quarter 2015 .

[30] Y. A. Chau and S. H. Yu, "Space modulation on wireless fading channels," in Proc. of IEEE 54th VTC (Fall), Sep. 2001, pp. 1668-1671.

[31] J. Jeganathan, A. Ghrayeb, L. Szczecinski, and A. Ceron, "Space shift keying modulation for MIMO channels," IEEE Transactions on Wireless Communications, vol. 8, no. 7, pp. 3692-3703, 2009.

[32] S. Ganesan, R. Mesleh, and et.al, "On the performance of spatial modulation of OFDM," in Proc. 40th Asilomar Conf. Sig., Syst. Comput., Oct. 29 - Nov. 1 2006, pp. 1825-1829.

[33] J. Wang, S. Jia, and J. Song, "Generalised spatial modulation system with multiple active transmit antennas and low complexity detection scheme," IEEE Trans. Wirel. Commun., vol. 11, no. 4, pp. 1605-1615, Apr. 2012.

[34] S. Sugiura, S. Chen, and L. Hanzo, "Coherent and differential spacetime shift keying: A dispersion matrix approach," IEEE Transactions on Communications, vol. 58, no. 11, pp. 3219 - 3230, November 2010.

[35] L.-L. Yang, "Transmitter preprocessing aided spatial modulation for multiple-input multiple-output systems," in Proc. of IEEE 73th VTC (Spring), May 2011.

[36] R. Zhang, L. L. Yang, and L. Hanzo, "Generalised pre-coding aided spatial modulation," IEEE Transactions on Wireless Communications, vol. 12 , no. 11 , pp. 5434-5443, November 2013.

[37] N. Serafimovski, S. Sinanovï̈Ğ, A. Younis, M. D. Renzo, and H. Haas, "2-user multiple access spatial modulation," in 2011 IEEE GLOBECOM Workshops (GC Wkshps), Dec 2011, pp. 343-347.

[38] L.-L. Yang, "Signal detection in antenna-hopping space-division multiple-access systems with space-shift keying modulation," IEEE Trans. Sig. Process., vol. 60, no. 1, pp. 351-366, Jan. 2012.

[39] M. D. Renzo and H. Haas, "Bit error probability of space-shift keying MIMO over multiple-access independent fading channels," IEEE Transactions on Vehicular Technology, vol. 60, no. 8, pp. 3694-3711, Oct 2011.

[40] A. Garcia-Rodriguez and C. Masouros, "Low-complexity compressive sensing detection for spatial modulation in large-scale multiple access channels," IEEE Transactions on Communications, vol. 63, no. 7, pp. 2565-2579, July 2015 
[41] L. He, J. Wang, J. Song, and L. Hanzo, "Bandwidth efficiency maximization for single-cell massive spatial modulation MIMO: An adaptive power allocation perspective," IEEE Access, vol. 5, pp. 1482-1495, 2017.

[42] — - "On the multi-user multi-cell massive spatial modulation uplink: How many antennas for each user?" IEEE Transactions on Wireless Communications, vol. 16, no. 3, pp. 1437-1451, March 2017.

[43] J. Zheng, "Low-complexity detector for spatial modulation multiple access channels with a large number of receive antennas," IEEE Communications Letters, vol. 18, no. 11, pp. 2055-2058, Nov 2014.

[44] D. SinanoviÄĞ, G. ĂăiÅąul, and B. Modlic, "Low-PAPR spatial modulation for SC-FDMA," IEEE Transactions on Vehicular Technology, vol. 66, no. 1, pp. 443-454, Jan 2017.

[45] M. Fukuma and K. Ishii, "Space-time code division multiple access based on spatial modulation," in 2015 IEEE 82nd Vehicular Technology Conference (VTC2015-Fall), Sept 2015, pp. 1-5.

[46] M. I. Kadir, S. Sugiura, J. Zhang, S. Chen, and L. Hanzo, "OFDMA/SCFDMA aided space-time shift keying for dispersive multiuser scenarios," IEEE Transactions on Vehicular Technology, vol. 62, no. 1, pp. 408-414, Jan 2013.

[47] J. Bao, et.al, "Joint multiuser detection of multidimensional constellations over fading channels," IEEE Transactions on Communications, vol. 65, no. 1, pp. 161-172, Jan. 2017.

[48] D. Cai, P. Fan and P. T. Mathiopoulos, "A tight lower bound for the symbol error performance of the uplink sparse code multiple access," IEEE Wireless Comm. Letters, vol. 6, no. 2, pp. 190-193, Apr. 2017.

[49] A. Graham, "Kronecker products and matrix calculus: With applications." John Wiley \& Sons, Inc., New York, 1982

[50] R. Gallager, "Low-density parity-check codes," IRE Transactions on information theory, vol. 8, no. 1, pp. 21-28, 1962.

[51] M. C. Davey and D. MacKay, "Low-density parity check codes over GF(q)," IEEE Communications Letters, vol. 2, no. 6, pp. 165-167, June 1998.

[52] W. E. Ryan and S. Lin, Channel Codes: Classical and Modern. New York: Cambridge University Press, 2009.

[53] M. D. Renzo and H. Haas, "Bit error probability of SM-MIMO over generalized fading channels," IEEE Transactions on Vehicular Technology, vol. 61, no. 3, pp. 1124-1144, March 2012.

[54] M. K. Simon and M.-S. Alouini, Digital Communication over Fading Channels, 2nd ed. John Wiley \& Sons, 2005.

[55] J. G. Proakis, Digital Communications, 5th ed. McGraw Hill, 2007.

[56] L.-L. Yang, Multicarrier Communications. Chichester, United Kingdom: John Wiley, 2009.

[57] M.-S. Alouini and A. J. Goldsmith, "A unified approach for calculating error rates of linearly modulated signals over generalized fading channels," IEEE Transactions on Communications, vol. 47, no. 9, pp. 1324-1334, September 1999.

[58] L.-L. Yang and L. Hanzo, "Performance of generalized multicarrie DS-CDMA over Nakagami- $m$ fading channels," IEEE Transactions on Communications, vol. 50, no. 6, pp. 956 - 966, June 2002.

[59] I. Gradshteyn and I. Ryzhik, Table of Integrals, Series, and Products, 7th ed. New York: Academic Press, Inc, 2007.

[60] L. Hanzo, S. X. Ng, W. Webb, and T. Keller, Quadrature amplitude modulation: From basics to adaptive trellis-coded, turbo-equalised and space-time coded OFDM, CDMA and MC-CDMA systems. IEEE PressJohn Wiley, 2004

[61] A. J. Goldsmith and S.-G. Chua, "Variable-rate variable-power MQAM for fading channels," IEEE Transactions on communications, vol. 45 , no. 10 , pp. $1218-1230,1997$.

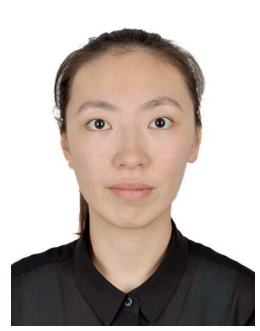

Yusha Liu received Her BEng degree in Communication Engineering from Xiamen University, China, and her MSc degree in wireless communications from the University of Southampton, UK, where she is currently pursuing her $\mathrm{PhD}$ degree with the Southampton Wireless Group. Her research interests include spatial modulation, non-orthogonal mulitiple access (NOMA) and compressive sensing for wireless communications.

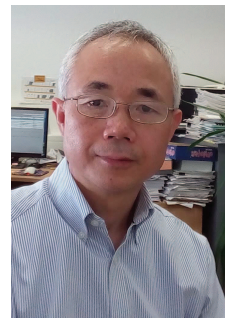

Lie-Liang Yang (M'98, SM'02, F'16) received his BEng degree in communications engineering from Shanghai TieDao University, Shanghai, China in 1988, and his MEng and $\mathrm{PhD}$ degrees in communications and electronics from Northern (Beijing) Jiaotong University, Beijing, China in 1991 and 1997, respectively. From June 1997 to December 1997 he was a visiting scientist to the Institute of Radio Engineering and Electronics, Academy of Sciences of the Czech Republic. Since December 1997, he has been with the University of Southampton, United Kingdom, where he is the professor of wireless communications in the School of Electronics and Computer Science. He has research interest in wireless communications, wireless networks and signal processing for wireless communications, as well as molecular communications and nano-networks. He has published over 350 research papers in journals and conference proceedings, authored/co-authored three books and also published several book chapters. The details about his research publications can be found at http://www.mobile.ecs.soton.ac.uk/lly/. He is a fellow of both the IEEE and the IET, and a distinguished lecturer of the IEEE. He served as an associate editor to the IEEE Trans. on Vehicular Technology and Journal of Communications and Networks (JCN), and is currently an associate editor to the IEEE Access.

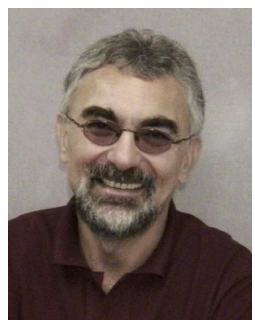

Lajos Hanzo (http://www-mobile.ecs.soton.ac.uk) FREng, FIEEE, FIET, Fellow of EURASIP, DSc received his degree in electronics in 1976 and his doctorate in 1983. In 2009 he was awarded an honorary doctorate by the Technical University of Budapest and in 2015 by the University of Edinburgh. In 2016 he was admitted to the Hungarian Academy of Science. During his 40-year career in telecommunications he has held various research and academic posts in Hungary, Germany and the UK. Since 1986 he has been with the School of Electronics and Computer Science, University of Southampton, UK, where he holds the chair in telecommunications. He has successfully supervised 111 PhD students, co-authored 18 John Wiley/IEEE Press books on mobile radio communications totalling in excess of 10000 pages, published 1701 research contributions at IEEE Xplore, acted both as TPC and General Chair of IEEE conferences, presented keynote lectures and has been awarded a number of distinctions. Currently he is directing a 60-strong academic research team, working on a range of research projects in the field of wireless multimedia communications sponsored by industry, the Engineering and Physical Sciences Research Council (EPSRC) UK, the European Research Council's Advanced Fellow Grant and the Royal Society's Wolfson Research Merit Award. He is an enthusiastic supporter of industrial and academic liaison and he offers a range of industrial courses. He is also a Governor of the IEEE VTS. During 2008 - 2012 he was the Editor-in-Chief of the IEEE Press and a Chaired Professor also at Tsinghua University, Beijing. For further information on research in progress and associated publications please refer to http://wwwmobile.ecs.soton.ac.uk Lajos has $30000+$ citations and an H-index of 70 